\title{
ASYMPTOTIC BEHAVIOR OF THE NAVIER-STOKES SYSTEM IN A THIN DOMAIN WITH NAVIER CONDITION ON A SLIGHTLY ROUGH BOUNDARY*
}

\author{
J. CASADO-DÍAZ ${ }^{\dagger}$, M. LUNA-LAYNEZ ${ }^{\dagger}$, AND F. J. SUÁREZ-GRAU ${ }^{\dagger}$
}

\begin{abstract}
We study the asymptotic behavior of the solutions of the Navier-Stokes system in a thin domain $\Omega_{\varepsilon}$ of thickness $\varepsilon$ satisfying the Navier boundary condition on a periodic rough set $\Gamma_{\varepsilon} \subset \partial \Omega_{\varepsilon}$ of period $r_{\varepsilon}$ and amplitude $\delta_{\varepsilon}$, with $\delta_{\varepsilon} \ll r_{\varepsilon} \ll \varepsilon$. We prove that the limit behavior as $\varepsilon$ goes to zero depends on the limit $\lambda$ of $\delta_{\varepsilon} \varepsilon^{\frac{1}{2}} / r_{\varepsilon}^{\frac{3}{2}}$. Namely, if $\lambda=+\infty$, the roughness is so strong that the fluid behaves as if we had imposed the adherence condition on $\Gamma_{\varepsilon}$. If $\lambda=0$, the roughness is too weak and the fluid behaves as if $\Gamma_{\varepsilon}$ were a plane. Finally, if $\lambda \in(0,+\infty)$, the roughness is strong enough to make a new friction term appear in the limit.
\end{abstract}

Key words. Navier-Stokes equations, Navier condition, rough boundary, thin fluid films

AMS subject classifications. 76A20, 35B27, 35Q30

DOI. $10.1137 / 120873479$

1. Introduction. The most usual boundary condition for a viscous fluid surrounded by an impermeable wall is the adherence condition, which establishes that the velocity of the fluid vanishes on the boundary. However, some other boundary conditions can be imposed. The Navier boundary condition consists in adding to the impenetrability condition (i.e., that the normal velocity vanishes) that, on the tangential component, the wall acts as a friction force. In the present paper we are interested in the relationship between the adherence and the Navier conditions for the case of a rough boundary.

In [12], it has been considered a rough boundary $\Gamma_{\varepsilon}$ described by the equation

$$
x_{3}=r_{\varepsilon} \Psi\left(\frac{x_{1}}{r_{\varepsilon}}, \frac{x_{2}}{r_{\varepsilon}}\right) \quad \forall\left(x_{1}, x_{2}\right) \in \omega,
$$

with $r_{\varepsilon}>0$ a parameter devoted to converge to zero, $\omega$ a bounded open set of $\mathbb{R}^{2}$, and $\Psi$ a smooth periodic function. It has been proved that, assuming that the wall $\Gamma_{\varepsilon}$ is impermeable (i.e., the normal velocity vanishes) and that the velocity is bounded in the topology of the Sobolev space $H^{1}$, then, in the limit, the velocity $u$ of the fluid satisfies the condition

$$
u\left(x_{1}, x_{2}, 0\right) \nabla \Psi\left(z_{1}, z_{2}\right)=0 \quad \forall\left(z_{1}, z_{2}\right) \in \mathbb{R}^{2} \text {, a.e. }\left(x_{1}, x_{2}\right) \in \omega .
$$

In particular, if

$$
\operatorname{Span}\left(\left\{\nabla \Psi\left(z_{1}, z_{2}\right):\left(z_{1}, z_{2}\right) \in \mathbb{R}^{2}\right\}\right)=\mathbb{R}^{2},
$$

which always holds except in the case where $\Psi$ is constant in one direction, we get that the velocity of the fluid vanishes on the boundary; i.e., it satisfies the adherence

\footnotetext{
* Received by the editors April 13, 2012; accepted for publication (in revised form) April 1, 2013; published electronically May 30, 2013. This work was partially supported by project MTM201124457 of the "Ministerio de Economía y Competitividad" and project FQM309 of the "Junta de Andalucía."

http://www.siam.org/journals/sima/45-3/87347.html

†Departamento de Ecuaciones Diferenciales y Análisis Numérico, Facultad de Matemáticas, Universidad de Sevilla, 41012-Sevilla, Spain (jcasadod@us.es, mllaynez@us.es, fjsgrau@us.es).
}

1641 
condition. Therefore, under these assumptions, the Navier and adherence conditions are asymptotically equivalent. This shows that the adherence condition, which is usually observed in practice, may be due to the existence of microasperities.

The equivalence between the Navier and adherence conditions was also proved in [10] for the more general case of a boundary (not necessarily periodic) described by the equation

$$
x_{3}=\Psi_{\varepsilon}\left(x_{1}, x_{2}\right) \quad \forall\left(x_{1}, x_{2}\right) \in \omega,
$$

with $\Psi_{\varepsilon}$ a sequence of Lipschitz functions which converges uniformly to zero and such that the support of the Young measure associated to $\nabla \Psi_{\varepsilon}$ contains at least two independent vectors.

In [14] was considered the case of a viscous fluid satisfying the Navier condition on a slightly rough boundary described by the equation

$$
x_{3}=\Psi_{\varepsilon}\left(x_{1}, x_{2}\right)=\delta_{\varepsilon} \Psi\left(\frac{x_{1}}{r_{\varepsilon}}, \frac{x_{2}}{r_{\varepsilon}}\right) \quad \forall\left(x_{1}, x_{2}\right) \in \omega,
$$

with

$$
\lim _{\varepsilon \rightarrow 0} \frac{\delta_{\varepsilon}}{r_{\varepsilon}}=0
$$

and $\Psi$ smooth and periodic. Note that (1.3) implies that $\nabla \Psi_{\varepsilon}$ converges uniformly to zero, and so the Young measure associated to $\nabla \Psi_{\varepsilon}$ is zero. Therefore we are not in the conditions of [10]. It was proved in [14] (see also [16]) that now the asymptotic behavior of the fluid depends on the limit

$$
\tilde{\lambda}=\lim _{\varepsilon \rightarrow 0} \frac{\delta_{\varepsilon}}{r_{\varepsilon}^{3 / 2}} .
$$

Namely, if $\delta_{\varepsilon} / r_{\varepsilon}^{\frac{3}{2}}$ tends to infinity and (1.2) holds, then the Navier and adherence boundary conditions are still asymptotically equivalent, while if $\delta_{\varepsilon} / r_{\varepsilon}^{\frac{3}{2}}$ tends to zero, then the fluid behaves as if the boundary were a plane. The case $\delta_{\varepsilon} \sim r_{\varepsilon}^{\frac{3}{2}}$ is the critical size where the roughness is not so large to imply the adherence condition but is large enough to make a new friction term appear in the limit.

A general result about the form of the limit equation for the Navier-Stokes system satisfying the Navier condition on a (nonnecessarily periodic) rough boundary has been obtained in [9].

The above results relate to a fixed height domain. Our aim in the present paper is to extend the results in [14] to the case of a domain of small height $\varepsilon$. Namely, for a smooth bounded open set $\omega \subset \mathbb{R}^{2}$ and a function $\Psi$ in $W_{\text {loc }}^{2, \infty}\left(\mathbb{R}^{2}\right)$, periodic of period $Z^{\prime}=(-1 / 2,1 / 2)^{2}$, we will consider the open set $\Omega_{\varepsilon}$ given by

$$
\Omega_{\varepsilon}=\left\{\left(x_{1}, x_{2}, x_{3}\right) \in \omega \times \mathbb{R}:-\delta_{\varepsilon} \Psi\left(\frac{x_{1}}{r_{\varepsilon}}, \frac{x_{2}}{r_{\varepsilon}}\right)<x_{3}<\varepsilon\right\} .
$$

The parameters $r_{\varepsilon}, \delta_{\varepsilon}$ are positive and satisfy $\delta_{\varepsilon} \ll r_{\varepsilon} \ll \varepsilon$ in the following sense:

$$
\lim _{\varepsilon \rightarrow 0} \frac{r_{\varepsilon}}{\varepsilon}=0, \quad \lim _{\varepsilon \rightarrow 0} \frac{\delta_{\varepsilon}}{r_{\varepsilon}}=0 .
$$

Copyright $@$ by SIAM. Unauthorized reproduction of this article is prohibited. 
Assuming a viscous fluid governed by the Navier-Stokes system and satisfying the Navier condition on the rough boundary

$$
\Gamma_{\varepsilon}=\left\{\left(x_{1}, x_{2}, x_{3}\right) \in \omega \times \mathbb{R}: x_{3}=-\delta_{\varepsilon} \Psi\left(\frac{x_{1}}{r_{\varepsilon}}, \frac{x_{2}}{r_{\varepsilon}}\right)\right\},
$$

we show that its pressure and velocity converge to the solutions of a Reynolds system which depends on

$$
\lambda=\lim _{\varepsilon \rightarrow 0} \frac{\delta_{\varepsilon}}{r_{\varepsilon}^{3 / 2}} \varepsilon^{1 / 2} .
$$

The role of $\lambda$ is similar to that of the limit $\tilde{\lambda}$ defined by (1.4); in fact these parameters agree if the height $\varepsilon$ is tending to one. Thus, we have the following:

- If $\lambda=\infty$ and (1.2) holds, then the fluid behaves as if we imposed an adherence condition.

- If $\lambda \in(0,+\infty)$, then the roughness is not strong enough to give the adherence condition in the limit but is enough to obtain a new friction term in the limit.

- If $\lambda=0$, the roughness is so weak that the fluid behaves as if the wall were a plane.

As in [14], the proof of our results is based on the unfolding method [4], [11], [18], but here it is necessary to combine it with a rescaling in the height variable, in order to work with a domain of height one. Our results were announced in [15] for the case of the Stokes system.

The above references given in this introduction are related to the asymptotic behavior of a viscous fluid satisfying the Navier condition on a rough boundary. Other boundary conditions have been considered by other authors. For example, the case of nonhomogeneous Dirichlet conditions is studied in [2], and [19] for fixed height domains, and in [5], [6], [7], and [8] for small height domains. The case of fixed height domains with Fourier conditions is considered in [3].

2. Notation. The elements $x \in \mathbb{R}^{3}$ will be decomposed as $x=\left(x^{\prime}, x_{3}\right)$ with $x^{\prime} \in \mathbb{R}^{2}, x_{3} \in \mathbb{R}$.

By $Z^{\prime}$, we denote the unitary cube of $\mathbb{R}^{2}, Z^{\prime}=\left(-\frac{1}{2}, \frac{1}{2}\right)^{2}$, and by $\widehat{Q}$ the set $\widehat{Q}=Z^{\prime} \times(0,+\infty)$. For every $M>0$ we write $\widehat{Q}_{M}=Z^{\prime} \times(0, M)$.

We use the index \# to mean periodicity with respect $Z^{\prime}$; for example, $L_{\#}^{2}\left(Z^{\prime}\right)$ denotes the space of functions $u \in L_{\text {loc }}^{2}\left(\mathbb{R}^{2}\right)$ which are $Z^{\prime}$-periodic, while $L_{\#}^{2}(\widehat{Q})$ denotes the space of functions $\widehat{u} \in L_{l o c}^{2}\left(\mathbb{R}^{2} \times(0,+\infty)\right)$ such that

$$
\int_{\widehat{Q}}|\widehat{u}|^{2} d z<+\infty, \quad \widehat{u}\left(z^{\prime}+k^{\prime}, z_{3}\right)=\widehat{u}(z) \quad \forall k^{\prime} \in \mathbb{Z}^{2}, \quad \text { a.e. } z \in \mathbb{R}^{2} \times(0,+\infty) .
$$

For a bounded measurable set $\Theta \subset \mathbb{R}^{N}$, we denote by $L_{0}^{2}(\Theta)$ the space of functions of $L^{2}(\Theta)$ with null integral. satisfy

We denote by $\varepsilon, r_{\varepsilon}$, and $\delta_{\varepsilon}$ three positive parameters which tend to zero and

$$
\lim _{\varepsilon \rightarrow 0} \frac{\delta_{\varepsilon}}{r_{\varepsilon}}=0, \quad \lim _{\varepsilon \rightarrow 0} \frac{r_{\varepsilon}}{\varepsilon}=0 .
$$

Then, for a function $\Psi \in W_{\#}^{2, \infty}\left(Z^{\prime}\right), \Psi \geq 0$ in $Z^{\prime}$, we define the open set $\Lambda_{\varepsilon} \subset \mathbb{R}^{3}$ by

$$
\Lambda_{\varepsilon}=\left\{x \in \mathbb{R}^{3}:-\delta_{\varepsilon} \Psi\left(\frac{x^{\prime}}{r_{\varepsilon}}\right)<x_{3}<\varepsilon\right\}
$$

Copyright $@$ by SIAM. Unauthorized reproduction of this article is prohibited. 
and for a smooth connected open set $\omega \subset \mathbb{R}^{2}$, we take

$$
\begin{aligned}
\Omega_{\varepsilon} & =\Lambda_{\varepsilon} \cap(\omega \times \mathbb{R}), \\
\Omega_{\varepsilon}^{-} & =\Omega_{\varepsilon} \cap(\omega \times(-\infty, 0)), \quad \Omega_{\varepsilon}^{+}=\Omega_{\varepsilon} \cap(\omega \times(0,+\infty)), \\
\Gamma_{\varepsilon} & =\left\{x \in \mathbb{R}^{3}: x^{\prime} \in \omega, x_{3}=-\delta_{\varepsilon} \Psi\left(\frac{x^{\prime}}{r_{\varepsilon}}\right)\right\}, \\
\widetilde{\Omega}_{\varepsilon} & =\left\{y \in \mathbb{R}^{3}: y^{\prime} \in \omega,-\frac{\delta_{\varepsilon}}{\varepsilon} \Psi\left(\frac{y^{\prime}}{r_{\varepsilon}}\right)<y_{3}<1\right\}, \\
\widetilde{\Gamma}_{\varepsilon} & =\left\{y \in \mathbb{R}^{3}: y^{\prime} \in \omega, y_{3}=-\frac{\delta_{\varepsilon}}{\varepsilon} \Psi\left(\frac{y^{\prime}}{r_{\varepsilon}}\right)\right\}, \\
\Omega & =\omega \times(0,1), \quad \Gamma=\omega \times\{0\} .
\end{aligned}
$$

We denote by $\nu$ the outside unitary normal vector to $\Omega_{\varepsilon}$ on $\partial \Omega_{\varepsilon}$.

The orthogonal projection on the tangent space of $\partial \Omega_{\varepsilon}$ will be denoted by $T$, i.e.,

$$
T \xi=\xi-(\xi \nu) \nu \quad \forall \xi \in \mathbb{R}^{3}, \text { a.e. on } \partial \Omega_{\varepsilon} .
$$

For $k^{\prime} \in \mathbb{Z}^{2}$ and $\rho>0$, we denote

$$
C_{\rho}^{k^{\prime}}=\rho k^{\prime}+\rho Z^{\prime}, \quad Q_{\rho}^{k^{\prime}}=\Lambda_{\varepsilon} \cap\left(C_{\rho}^{k^{\prime}} \times \mathbb{R}\right) .
$$

We define $\kappa: \mathbb{R}^{2} \rightarrow \mathbb{Z}^{2}$ by

$$
\kappa\left(x^{\prime}\right)=k^{\prime} \Leftrightarrow x^{\prime} \in C_{1}^{k^{\prime}} .
$$

Note that $\kappa$ is well defined up to a set of zero measure in $\mathbb{R}^{2}$ (the set $\cup_{k^{\prime} \in \mathbb{Z}^{2}} \partial C_{1}^{k^{\prime}}$ ). Moreover, for every $\rho>0$, we have

$$
\kappa\left(\frac{x^{\prime}}{\rho}\right)=k^{\prime} \Leftrightarrow x^{\prime} \in C_{\rho}^{k^{\prime}} .
$$

For a.e. $x^{\prime} \in \mathbb{R}^{2}$ we define $C_{r_{\varepsilon}}\left(x^{\prime}\right)$ as the square $C_{r_{\varepsilon}}^{k^{\prime}}$ such that $x^{\prime}$ belongs to $C_{r_{\varepsilon}}^{k^{\prime}}$. We denote by $\mathcal{V}$ the space of functions $\widehat{v}: \mathbb{R}^{2} \times(0,+\infty) \rightarrow \mathbb{R}$ such that

$$
\widehat{v} \in H_{\#}^{1}\left(\widehat{Q}_{M}\right) \quad \forall M>0, \quad \nabla \widehat{v} \in L_{\#}^{2}(\widehat{Q})^{3} .
$$

It is a Hilbert space endowed with the norm $\|\cdot\|_{\mathcal{V}}$ defined by

$$
\|\widehat{v}\|_{\mathcal{V}}^{2}=\|\widehat{v}\|_{L^{2}\left(Z^{\prime} \times\{0\}\right)}^{2}+\|\nabla \widehat{v}\|_{L^{2}(\widehat{Q})^{3}}^{2} .
$$

We denote by $O_{\varepsilon}$ a generic real sequence which tends to zero with $\varepsilon$ and can change from line to line.

We denote by $C$ a generic positive constant which can change from line to line.

3. Main results. In the present section we describe the asymptotic behavior of a sequence $\left(u_{\varepsilon}, p_{\varepsilon}\right)$, solution of the Navier-Stokes system

$$
\left\{\begin{array}{l}
-\mu \Delta u_{\varepsilon}+\left(u_{\varepsilon} \cdot \nabla\right) u_{\varepsilon}+\nabla p_{\varepsilon}=f_{\varepsilon} \text { in } \Omega_{\varepsilon} \\
\operatorname{div} u_{\varepsilon}=0 \text { in } \Omega_{\varepsilon} \\
u_{\varepsilon} \nu=0, \quad T\left(\mu \frac{\partial u_{\varepsilon}}{\partial \nu}+\frac{\gamma}{\varepsilon} u_{\varepsilon}\right)=0 \text { on } \Gamma_{\varepsilon} \\
u_{\varepsilon}=0 \text { on } \partial \Omega_{\varepsilon} \backslash \Gamma_{\varepsilon}
\end{array}\right.
$$

Copyright $@$ by SIAM. Unauthorized reproduction of this article is prohibited. 
where $\Omega_{\varepsilon}$ and $\Gamma_{\varepsilon}$ are defined by (2.3) and (2.5). The viscosity $\mu$ is assumed strictly positive and the friction coefficient $\gamma$ nonnegative. The right-hand side $f_{\varepsilon}$ is of the form

$$
f_{\varepsilon}(x)=\tilde{f}\left(x^{\prime}, \frac{x_{3}}{\varepsilon}\right), \quad \text { a.e. } x \in \Omega_{\varepsilon},
$$

where $\tilde{f}$ is assumed in $L^{2}(\omega \times(-1,1))^{3}$. The proof of the corresponding results will be given in the following sections.

The existence of solution for problem (3.1) and a priori estimate is given by the following result.

THEOREM 3.1. We consider $\omega$ a bounded domain of class $C^{2}$. Then problem (3.1) has at least a solution $\left(u_{\varepsilon}, p_{\varepsilon}\right)$ in $H^{1}\left(\Omega_{\varepsilon}\right)^{3} \times L_{0}^{2}\left(\Omega_{\varepsilon}\right)$. Moreover, there exist $\varepsilon_{0}>0$ and $C>0$ such that for $\varepsilon \in\left(0, \varepsilon_{0}\right)$, every solution $\left(u_{\varepsilon}, p_{\varepsilon}\right)$ of (3.1) satisfies

$$
\left\|u_{\varepsilon}\right\|_{L^{2}\left(\Omega_{\varepsilon}\right)^{3}} \leq C \varepsilon^{5 / 2}, \quad\left\|D u_{\varepsilon}\right\|_{L^{2}\left(\Omega_{\varepsilon}\right)^{3 \times 3}} \leq C \varepsilon^{3 / 2}, \quad\left\|p_{\varepsilon}\right\|_{L_{0}^{2}\left(\Omega_{\varepsilon}\right)} \leq C \varepsilon^{1 / 2} .
$$

In fact, the pressure $p_{\varepsilon}$ can be decomposed as $p_{\varepsilon}=p_{\varepsilon}^{0}+p_{\varepsilon}^{1}$, with $p_{\varepsilon}^{0} \in H^{1}(\omega)$ (it does not depend on the variable $\left.x_{3}\right), p_{\varepsilon}^{1} \in L^{2}\left(\Omega_{\varepsilon}\right)$, and

$$
\left\|p_{\varepsilon}^{0}\right\|_{H^{1}(\omega)} \leq C, \quad\left\|p_{\varepsilon}^{1}\right\|_{L^{2}\left(\Omega_{\varepsilon}\right)} \leq C \varepsilon^{3 / 2} .
$$

Remark 3.2. Although we have stated Theorem 3.1 for $\omega$ of class $C^{2}$, the result can be extended for $\omega$ Lipschitz or more generally for an open set satisfying the interior uniform cone condition, but the proof is much more difficult. This corresponds to a particular case of a work in progress, where we also give some applications to linear elasticity. This would extend Theorems $3.3,3.4$, and 3.8 below to the case of $\omega$ Lipschitz.

As is usual when dealing with thin domains, we use the dilatation

$$
y^{\prime}=x^{\prime}, \quad y_{3}=\frac{x_{3}}{\varepsilon},
$$

which transforms $\Omega_{\varepsilon}$ in the sequence of open sets with fixed height, $\widetilde{\Omega}_{\varepsilon}$, defined by (2.6). Thus, we introduce $\tilde{u}_{\varepsilon} \in H^{1}\left(\widetilde{\Omega}_{\varepsilon}\right)^{3}$, and $\tilde{p}_{\varepsilon} \in L_{0}^{2}\left(\widetilde{\Omega}_{\varepsilon}\right)$ by

$$
\tilde{u}_{\varepsilon}(y)=u_{\varepsilon}\left(y^{\prime}, \varepsilon y_{3}\right), \quad \tilde{p}_{\varepsilon}(y)=p_{\varepsilon}\left(y^{\prime}, \varepsilon y_{3}\right), \quad \text { a.e. } y \in \widetilde{\Omega}_{\varepsilon} .
$$

Our goal then is to describe the asymptotic behavior of these new sequences $\tilde{u}_{\varepsilon}$, $\tilde{p}_{\varepsilon}$. This is given by the following theorem.

Theorem 3.3. We consider $\omega$ a bounded domain of class $C^{2}$. Assume there exists (this always holds for a subsequence)

$$
\lambda=\lim _{\varepsilon \rightarrow 0} \frac{\delta_{\varepsilon} \varepsilon^{\frac{1}{2}}}{r_{\varepsilon}^{\frac{3}{2}}} \in[0,+\infty] .
$$

Let $\left(u_{\varepsilon}, p_{\varepsilon}\right) \in H^{1}\left(\Omega_{\varepsilon}\right)^{3} \times L_{0}^{2}\left(\Omega_{\varepsilon}\right)$ be a solution of (3.1) and let $\tilde{u}_{\varepsilon}, \tilde{p}_{\varepsilon}$ be given by (3.6). Then we have

$\frac{\tilde{u}_{\varepsilon}}{\varepsilon} \rightarrow 0$ in $H^{1}(\Omega)^{3}, \frac{\tilde{u}_{\varepsilon}}{\varepsilon^{2}} \rightarrow\left(\tilde{u}^{\prime}, 0\right)$ in $H^{1}\left(0,1 ; L^{2}(\omega)\right)^{3}, \frac{\tilde{u}_{\varepsilon, 3}}{\varepsilon^{3}} \rightarrow \tilde{w}$ in $H^{2}\left(0,1 ; H^{-1}(\omega)\right)$,

$$
\tilde{p}_{\varepsilon} \rightarrow \tilde{p} \text { in } L^{2}(\Omega), \quad \frac{\partial_{y_{3}} \tilde{p}_{\varepsilon}}{\varepsilon} \rightarrow \tilde{f}_{3} \text { in } L^{2}\left(\omega ; H^{-1}(0,1)\right),
$$

Copyright $@$ by SIAM. Unauthorized reproduction of this article is prohibited. 
where $\tilde{u}^{\prime} \in H^{1}\left(0,1 ; L^{2}(\omega)\right)^{2}, \tilde{w} \in H^{2}\left(0,1 ; H^{-1}(\omega)\right)$, and $\tilde{p} \in H^{1}(\omega) \cap L_{0}^{2}(\omega)$ are the unique solutions of the system

$$
\left\{\begin{array}{l}
-\mu \partial_{y_{3} y_{3}}^{2} \tilde{u}^{\prime}+\nabla_{y^{\prime}} \tilde{p}=\tilde{f}^{\prime} \quad \text { in } \Omega, \\
\operatorname{div}_{y^{\prime}} \tilde{u}^{\prime}+\partial_{y_{3}} \tilde{w}=0 \quad \text { in } \Omega \\
\tilde{u}^{\prime}\left(y^{\prime}, 1\right)=\tilde{w}\left(y^{\prime}, 0\right)=\tilde{w}\left(y^{\prime}, 1\right)=0 \text { in } \omega, \quad \int_{0}^{1} \tilde{u}^{\prime}(y) d y_{3} \nu=0 \text { on } \partial \omega
\end{array}\right.
$$

plus the following boundary condition on $\Gamma$, which depends on the value of $\lambda$.

(i) If $\lambda=+\infty$, then defining

$$
W=\operatorname{Span}\left(\left\{\nabla \Psi\left(z^{\prime}\right): z^{\prime} \in Z^{\prime}\right\}\right)
$$

we have that $\tilde{u}^{\prime}$ satisfies

$$
-\mu \partial_{y_{3}} \tilde{u}^{\prime}+\gamma \tilde{u}^{\prime} \in W, \quad \tilde{u}^{\prime} \in W^{\perp} \quad \text { on } \Gamma .
$$

(ii) If $\lambda \in(0,+\infty)$, then defining $\left(\widehat{\phi}^{i}, \widehat{q}^{i}\right) \in \mathcal{V}^{3} \times L_{\#}^{2}\left(Z^{\prime} \times \mathbb{R}^{+}\right)$, $i=1,2$, as a solution of

$$
\left\{\begin{array}{l}
-\mu \Delta_{z} \widehat{\phi}^{i}+\nabla_{z} \widehat{q}^{i}=0 \text { in } \mathbb{R}^{2} \times \mathbb{R}^{+} \\
\operatorname{div}_{z} \widehat{\phi}^{i}=0 \text { in } \mathbb{R}^{2} \times \mathbb{R}^{+}, \\
\widehat{\phi}_{3}^{i}=\partial_{z_{i}} \Psi, \partial_{z_{3}}\left(\widehat{\phi}^{i}\right)^{\prime}=0 \quad \text { on } \mathbb{R}^{2} \times\{0\}
\end{array}\right.
$$

and $R \in \mathbb{R}^{2 \times 2}$ by

$$
R_{i j}=\mu \int_{Z^{\prime} \times(0,+\infty)} D_{z} \widehat{\phi}^{i}: D_{z} \widehat{\phi}^{j} d z \quad \forall i, j \in\{1,2\}
$$

we have that $\tilde{u}^{\prime}$ satisfies

$$
-\mu \partial_{y_{3}} \tilde{u}^{\prime}+\gamma \tilde{u}^{\prime}+\lambda^{2} R \tilde{u}^{\prime}=0 \quad \text { on } \Gamma .
$$

(iii) If $\lambda=0$, then we have that $\tilde{u}^{\prime}$ satisfies

$$
-\mu \partial_{y_{3}} \tilde{u}^{\prime}+\gamma \tilde{u}^{\prime}=0 \quad \text { on } \Gamma .
$$

From (3.10), (3.12), (3.15), and (3.16), as is usual in the asymptotic study of fluids in thin domains, we can prove that the limit pressure $\tilde{p}$ is a solution of a Reynolds problem and that the functions $\tilde{u}^{\prime}$ and $\tilde{w}$ can be explicitly obtained from $\tilde{p}$. This implies in particular that the system satisfied by $\tilde{u}^{\prime}, \tilde{w}$, and $\tilde{p}$ has a unique solution such as has been stated in Theorem 3.3. For the sake of simplicity, we just consider the case where $\tilde{f}^{\prime}$ does not depend on the variable $y_{3}$. Note that this assumption usually holds in the applications because $\Omega_{\varepsilon}$ is very thin, and so the variations in height of the exterior forces can be neglected.

TheOREM 3.4. We consider $\omega$ a bounded domain of class $C^{2}$. Let $\left(u_{\varepsilon}, p_{\varepsilon}\right) \in$ $H^{1}\left(\Omega_{\varepsilon}\right)^{3} \times L_{0}^{2}\left(\Omega_{\varepsilon}\right)$ be a solution of (3.1) where the function $\tilde{f}^{\prime}$ is assumed to not depend on $y_{3}$. Then the functions $\tilde{u}^{\prime}, \tilde{w}, \tilde{p}$ defined by Theorem 3.3 are given by the following:

Copyright $@$ by SIAM. Unauthorized reproduction of this article is prohibited. 
(i) If $\lambda=+\infty$, then $\tilde{p}$ is the solution of the Reynolds problem

$$
\left\{\begin{array}{l}
-\operatorname{div}_{y^{\prime}}\left(\left(\frac{1}{3} I+\left(1+\frac{\gamma}{\mu}\right)^{-1} P_{W^{\perp}}\right)\left(\nabla_{y^{\prime}} \tilde{p}-\tilde{f}^{\prime}\right)\right)=0 \text { in } \omega \\
\left(\left(\frac{1}{3} I+\left(1+\frac{\gamma}{\mu}\right)^{-1} P_{W^{\perp}}\right)\left(\nabla_{y^{\prime}} \tilde{p}-\tilde{f}^{\prime}\right)\right) \nu=0 \quad \text { on } \partial \omega
\end{array}\right.
$$

where $P_{W^{\perp}}$ denotes the orthogonal projection from $\mathbb{R}^{2}$ to $W^{\perp}$. Moreover, $\tilde{u}^{\prime}$ and $\tilde{w}$ are given by

$$
\begin{gathered}
\tilde{u}^{\prime}(y)=\frac{\left(y_{3}-1\right)}{2 \mu}\left(y_{3} I+\left(1+\frac{\gamma}{\mu}\right)^{-1} P_{W^{\perp}}\right)\left(\nabla_{y^{\prime}} \tilde{p}\left(y^{\prime}\right)-\tilde{f}^{\prime}\left(y^{\prime}\right)\right), \quad \text { a.e. } y \in \Omega, \\
\tilde{w}(y)=-\int_{0}^{y_{3}} \operatorname{div}_{y^{\prime}} \tilde{u}^{\prime}\left(y^{\prime}, s\right) d s, \quad \text { a.e. } y \in \Omega .
\end{gathered}
$$

(ii) If $\lambda \in(0,+\infty)$, then $\tilde{p}$ is the solution of the Reynolds problem

$$
\left\{\begin{array}{l}
-\operatorname{div}_{y^{\prime}}\left(\left(\frac{1}{3} I+\left(\left(1+\frac{\gamma}{\mu}\right) I+\frac{\lambda^{2}}{\mu} R\right)^{-1}\right)\left(\nabla_{y^{\prime}} \tilde{p}-\tilde{f}^{\prime}\right)\right)=0 \text { in } \omega \\
\left(\left(\frac{1}{3} I+\left(\left(1+\frac{\gamma}{\mu}\right) I+\frac{\lambda^{2}}{\mu} R\right)^{-1}\right)\left(\nabla_{y^{\prime}} \tilde{p}-\tilde{f}^{\prime}\right)\right) \nu=0 \quad \text { on } \partial \omega .
\end{array}\right.
$$

Moreover, $\tilde{u}^{\prime}$ is given by

$$
\tilde{u}^{\prime}(y)=\frac{\left(y_{3}-1\right)}{2 \mu}\left(y_{3} I+\left(\left(1+\frac{\gamma}{\mu}\right) I+\frac{\lambda^{2}}{\mu} R\right)^{-1}\right)\left(\nabla_{y^{\prime}} \tilde{p}\left(y^{\prime}\right)-\tilde{f}^{\prime}\left(y^{\prime}\right)\right),
$$

a.e. $y \in \Omega$, and $\tilde{w}$ is defined by (3.19).

(iii) If $\lambda=0$, then $\tilde{p}$ is the solution of the Reynolds problem

$$
\left\{\begin{array}{l}
\left.-\operatorname{div}_{y^{\prime}}\left(\left(\frac{1}{3}+\left(1+\frac{\gamma}{\mu}\right)^{-1}\right)\left(\nabla_{y^{\prime}} \tilde{p}-\tilde{f}^{\prime}\right)\right)\right)=0 \text { in } \omega \\
\left.\left(\left(\frac{1}{3}+\left(1+\frac{\gamma}{\mu}\right)^{-1}\right)\left(\nabla_{y^{\prime}} \tilde{p}-\tilde{f}^{\prime}\right)\right)\right) \nu=0 \text { on } \partial \omega
\end{array}\right.
$$

Moreover, $\tilde{u}^{\prime}$ is given by

$$
\tilde{u}^{\prime}(y)=\frac{1}{2 \mu}\left(y_{3}^{2}+\left(1+\frac{\gamma}{\mu}\right)^{-1}\right)\left(\nabla_{y^{\prime}} \tilde{p}\left(y^{\prime}\right)-\tilde{f}^{\prime}\left(y^{\prime}\right)\right), \text { a.e. } y \in \Omega,
$$

and $\tilde{w}$ is the null function.

Remark 3.5. For $\lambda=+\infty$, Theorem 3.3 shows that $u_{\varepsilon}, p_{\varepsilon}$ behave as if we had assumed in (3.1) that $\Gamma_{\varepsilon}$ were the plane boundary $\left\{x_{3}=0\right\}$ and that the boundary condition on $\Gamma_{\varepsilon}$ were

$$
-\mu \partial_{x_{3}} u_{\varepsilon}^{\prime}+\gamma u_{\varepsilon}^{\prime} \in W, \quad u_{\varepsilon} \in W^{\perp} \times\{0\} \quad \text { on } \Gamma_{\varepsilon} .
$$

Copyright (c) by SIAM. Unauthorized reproduction of this article is prohibited. 
In particular, if $W$ is $\mathbb{R}^{2}$ (which is true except if $\Psi$ is constant in one direction), we deduce that the Navier condition in (3.1) is asymptotically equivalent to the adherence condition $u_{\varepsilon}=0$ on $\Gamma_{\varepsilon}$.

For $\lambda \in(0,+\infty)$, Theorem 3.3 shows that the asymptotic behavior of $u_{\varepsilon}$ and $p_{\varepsilon}$ is the same as if $\Gamma_{\varepsilon}$ were the plane boundary $\left\{x_{3}=0\right\}$ and the boundary condition on $\Gamma_{\varepsilon}$ were

$$
u_{\varepsilon, 3}=0, \quad-\mu \partial_{x_{3}} u_{\varepsilon}^{\prime}+\gamma u_{\varepsilon}^{\prime}+\lambda^{2} R u_{\varepsilon}^{\prime}=0 \quad \text { on } \Gamma_{\varepsilon} ;
$$

i.e., although the roughness is not strong enough to deduce that the Navier condition on $\Gamma_{\varepsilon}$ is equivalent to (3.24), it is sufficient to provide the new friction term $\lambda^{2} R u_{\varepsilon}^{\prime}$ in (3.25).

For $\lambda=0$, the roughness is so weak that $u_{\varepsilon}$ and $p_{\varepsilon}$ behave as if $\Gamma_{\varepsilon}$ were the plane boundary $\left\{x_{3}=0\right\}$ and the boundary condition on $\Gamma_{\varepsilon}$ were

$$
u_{\varepsilon, 3}=0, \quad-\mu \partial_{x_{3}} u_{\varepsilon}^{\prime}+\gamma u_{\varepsilon}^{\prime}=0 \quad \text { on } \Gamma_{\varepsilon} .
$$

Remark 3.6. The critical size $\lambda \in(0,+\infty)$ can be considered as the general one. In fact, the cases $\lambda=0, \lambda=+\infty$ can be obtained from this one, taking the limit in (3.15) when $\lambda$ tends to zero and infinity, respectively.

Remark 3.7. Theorem 3.3 generalizes the result proved in [14] for a fluid with fixed height. In [14] the critical size is $\delta_{\varepsilon} \approx r_{\varepsilon}^{3 / 2}$, which agrees with the critical size in the present paper $\delta_{\varepsilon} \approx r_{\varepsilon}^{3 / 2} / \varepsilon^{1 / 2}$ when $\varepsilon=1$. Moreover, the functions $\widehat{\phi}^{i}$ and $\widehat{q}^{i}$ are the same functions which appear in [14] to describe the behavior of the velocity and the pressure near the rough boundary. We observe that in our case, the expression (3.7) for $\lambda$ depends not only on the parameters $\delta_{\varepsilon}, r_{\varepsilon}$ which define $\Gamma_{\varepsilon}$ but also on the height $\varepsilon$ of $\Omega_{\varepsilon}$. This is due to the fact that far from the rough boundary the behavior of the fluid is different from the corresponding one in [14].

The following theorem (corrector result) provides approximations of $u_{\varepsilon}, D u_{\varepsilon}$, and $p_{\varepsilon}$ in the strong topology of $L^{2}\left(\Omega_{\varepsilon}\right)$. The sets $\Omega_{\varepsilon}^{+}$and $\Omega_{\varepsilon}^{-}$are defined in (2.4).

TheOREm 3.8. We consider $\omega$ a bounded domain of class $C^{2}$. Let $\left(u_{\varepsilon}, p_{\varepsilon}\right) \in$ $H^{1}\left(\Omega_{\varepsilon}\right)^{3} \times L_{0}^{2}\left(\Omega_{\varepsilon}\right)$ be a solution of (3.1) and let $\tilde{u}_{\varepsilon}, \tilde{p}_{\varepsilon}$ be defined by (3.6). Then, depending on the value of $\lambda$, the functions $\tilde{u}^{\prime} \in H^{1}\left(0,1 ; L^{2}(\omega)\right)^{2}, \tilde{w} \in H^{2}\left(0,1 ; H^{-1}(\omega)\right)$, and $\tilde{p} \in L_{0}^{2}(\omega)$ given by Theorem 3.3 satisfy the following:

$$
\begin{aligned}
& \lim _{\varepsilon \rightarrow 0} \frac{1}{\varepsilon^{5}} \int_{\Omega_{\varepsilon}^{-}}\left|u_{\varepsilon}\right|^{2} d x=0, \quad \lim _{\varepsilon \rightarrow 0} \frac{1}{\varepsilon^{5}} \int_{\Omega_{\varepsilon}^{+}}\left(\left|u_{\varepsilon}^{\prime}-\varepsilon^{2} \tilde{u}^{\prime}\left(x^{\prime}, \frac{x_{3}}{\varepsilon}\right)\right|^{2}+\left|u_{\varepsilon, 3}\right|^{2}\right) d x=0, \\
& (3.28) \quad \lim _{\varepsilon \rightarrow 0} \frac{1}{\varepsilon} \int_{\Omega_{\varepsilon}^{-}}\left|p_{\varepsilon}\right|^{2} d x=0, \quad \lim _{\varepsilon \rightarrow 0} \frac{1}{\varepsilon} \int_{\Omega_{\varepsilon}^{+}}\left|p_{\varepsilon}-\tilde{p}\left(x^{\prime}\right)\right|^{2} d x=0 .
\end{aligned}
$$

(ii) If $\lambda=0$ or $+\infty$, then we have

$$
\begin{aligned}
& \lim _{\varepsilon \rightarrow 0} \frac{1}{\varepsilon^{3}} \int_{\Omega_{\varepsilon}^{-}}\left|D u_{\varepsilon}\right|^{2} d x=0, \\
& \lim _{\varepsilon \rightarrow 0} \frac{1}{\varepsilon^{3}} \int_{\Omega_{\varepsilon}^{+}}\left|D u_{\varepsilon}-\varepsilon \sum_{i=1}^{2} \partial_{y_{3}} \tilde{u}_{i}\left(x^{\prime}, \frac{x_{3}}{\varepsilon}\right) e_{i} \otimes e_{3}\right|^{2} d x=0 .
\end{aligned}
$$

Copyright $@$ by SIAM. Unauthorized reproduction of this article is prohibited. 
(iii) If $\lambda \in(0+\infty)$, taking $\widehat{\phi}^{i}, i=1,2$, as a solution of (3.13) and defining $\widehat{u}: \omega \times\left(\mathbb{R}^{2} \times \mathbb{R}^{+}\right) \rightarrow \mathbb{R}^{3}$ by

$$
\widehat{u}\left(x^{\prime}, z\right)=-\lambda\left(\tilde{u}_{1}\left(x^{\prime}, 0\right) \widehat{\phi}^{1}(z)+\tilde{u}_{2}\left(x^{\prime}, 0\right) \widehat{\phi}^{2}(z)\right)
$$

for a.e. $\left(x^{\prime}, z\right) \in \omega \times\left(\mathbb{R}^{2} \times \mathbb{R}^{+}\right)$, then we have

$$
\begin{aligned}
& \lim _{\varepsilon \rightarrow 0} \frac{1}{\varepsilon^{3}} \int_{\Omega_{\varepsilon}^{-}}\left|D u_{\varepsilon}\right|^{2} d x=0, \\
& \lim _{\varepsilon \rightarrow 0} \frac{1}{\varepsilon^{3}} \int_{\Omega_{\varepsilon}^{+}} \mid D u_{\varepsilon}-\varepsilon \sum_{i=1}^{2} \partial_{y_{3}} \tilde{u}_{i}\left(x^{\prime}, \frac{x_{3}}{\varepsilon}\right) e_{i} \otimes e_{3} \\
& \quad-\left.\frac{\varepsilon^{\frac{3}{2}}}{r_{\varepsilon}^{\frac{1}{2}}} \int_{C_{r_{\varepsilon}\left(x^{\prime}\right)}} D_{z} \widehat{u}\left(s^{\prime}, \frac{x}{r_{\varepsilon}}\right) d s^{\prime}\right|^{2} d x=0 .
\end{aligned}
$$

Remark 3.9. If we assume that $\tilde{u}^{\prime}$ belongs to $H^{1}(\Omega)^{2}$, then we can rewrite the last limit in (3.31) as

$$
\lim _{\varepsilon \rightarrow 0} \frac{1}{\varepsilon^{3}} \int_{\Omega_{\varepsilon}^{+}}\left|D u_{\varepsilon}-\varepsilon \sum_{i=1}^{2} \partial_{y_{3}} \tilde{u}_{i}\left(x^{\prime}, \frac{x_{3}}{\varepsilon}\right) e_{i} \otimes e_{3}-\frac{\varepsilon^{\frac{3}{2}}}{r_{\varepsilon}^{\frac{1}{2}}} D_{z} \widehat{u}\left(s^{\prime}, \frac{x}{r_{\varepsilon}}\right)\right|^{2} d x=0 .
$$

By Theorem 3.4, if $\tilde{f}^{\prime}$ does not depend on $y_{3}$, a sufficient condition to have $\tilde{u}^{\prime}$ in $H^{1}(\Omega)^{2}$ is to assume $\tilde{f}^{\prime}$ in $H^{1}(\omega)^{2}$. Indeed, in this case the Reynolds equation for $\tilde{p}$, (3.20), shows that $\tilde{p}$ is in $H^{1}(\omega)$, and then by $(3.21) \tilde{u}^{\prime}$ is in $H^{1}(\Omega)^{2}$.

4. Existence of solution and a priori estimates. Our goal in this section is the proof of Theorem 3.1. For this purpose we need some previous estimates which are given by Lemma 4.1, Corollaries 4.2 and 4.3, and Proposition 4.4 below.

Lemma 4.1. Let $\omega$ be a domain of class $C^{2}$ (not necessarily bounded) of $\mathbb{R}^{2}$ and consider $\Omega_{\varepsilon}$ defined by (2.3) where $\delta_{\varepsilon}$ and $r_{\varepsilon}$ satisfy (2.1). Then there exist $\varepsilon_{0}>0$ and $C>0$ such that for every $\varepsilon \in\left(0, \varepsilon_{0}\right)$ and every $p_{\varepsilon}$ in $L_{\text {loc }}^{2}\left(\Omega_{\varepsilon}\right)$, with $\nabla p_{\varepsilon} \in H^{-1}\left(\Omega_{\varepsilon}\right)^{3}$, there exist $p_{\varepsilon}^{0} \in H_{\text {loc }}^{1}(\omega)$ (it does not depend on $x_{3}$ ) and $p_{\varepsilon}^{1} \in L^{2}\left(\Omega_{\varepsilon}\right)$ satisfying

$$
\begin{gathered}
p_{\varepsilon}=p_{\varepsilon}^{0}+p_{\varepsilon}^{1} \quad \text { in } \Omega_{\varepsilon}, \\
\varepsilon^{\frac{3}{2}}\left\|\nabla p_{\varepsilon}^{0}\right\|_{L^{2}(\omega)^{2}}+\left\|p_{\varepsilon}^{1}\right\|_{L^{2}\left(\Omega_{\varepsilon}\right)} \leq C\left\|\nabla p_{\varepsilon}\right\|_{H^{-1}\left(\Omega_{\varepsilon}\right)^{3}} .
\end{gathered}
$$

Proof. We divide the proof in two steps.

Step 1. Let us first consider the case $\omega=\mathbb{R}^{2}$. Using the change of variables $y=x / \varepsilon$, which transforms $\Omega_{\varepsilon}$ into

$$
\check{\Omega}_{\varepsilon}=\left\{y=\left(y^{\prime}, y_{3}\right) \in \mathbb{R}^{3}:-\check{\delta}_{\varepsilon} \Psi\left(\frac{y^{\prime}}{\check{r}_{\varepsilon}}\right)<y_{3}<1\right\},
$$

with $\check{\delta}_{\varepsilon}=\delta_{\varepsilon} / \varepsilon, \check{r}_{\varepsilon}=r_{\varepsilon} / \varepsilon$, we get that, in this case, Lemma 4.1 is equivalent to showing that there exists $C>0$ such that for every $p_{\varepsilon} \in L_{l o c}^{2}\left(\check{\Omega}_{\varepsilon}\right)$ with $\nabla p_{\varepsilon} \in$ $H^{-1}\left(\check{\Omega}_{\varepsilon}\right)^{3}$, there exist $p_{\varepsilon}^{0} \in H_{l o c}^{1}\left(\mathbb{R}^{2}\right)$ and $p_{\varepsilon}^{1} \in L^{2}\left(\check{\Omega}_{\varepsilon}\right)$ satisfying

$$
\begin{gathered}
p_{\varepsilon}=p_{\varepsilon}^{0}+p_{\varepsilon}^{1} \quad \text { in } \check{\Omega}_{\varepsilon}, \\
\left\|\nabla p_{\varepsilon}^{0}\right\|_{L^{2}\left(\mathbb{R}^{2}\right)^{2}}+\left\|p_{\varepsilon}^{1}\right\|_{L^{2}\left(\check{\Omega}_{\varepsilon}\right)} \leq C\left\|\nabla p_{\varepsilon}\right\|_{H^{-1}\left(\check{\Omega}_{\varepsilon}\right)^{3}} .
\end{gathered}
$$

Copyright $@$ by SIAM. Unauthorized reproduction of this article is prohibited. 
We will use the following notation:

We take $e_{1}^{\prime}, e_{2}^{\prime}$ to be the vectors of the canonical basis in $\mathbb{R}^{2}$.

For every $k^{\prime} \in \mathbb{Z}^{2}$ we define the sets $C_{k^{\prime}}$ and $\widetilde{C}_{k^{\prime}}$ by

$$
C_{k^{\prime}}=\left(k^{\prime}+Z^{\prime}\right) \times(0,1), \quad \check{C}_{k^{\prime}}=\left[\left(k^{\prime}+3 Z^{\prime}\right) \times \mathbb{R}\right] \cap \check{\Omega}_{\varepsilon},
$$

the sets $T_{k^{\prime}}^{+}, T_{k^{\prime}}^{-}$as the triangles in $\mathbb{R}^{2}$ with vertices $k^{\prime}, k^{\prime}+e_{1}^{\prime}, k^{\prime}+e_{2}^{\prime}$ and $k^{\prime}, k^{\prime}-$ $e_{1}^{\prime}, k^{\prime}-e_{2}^{\prime}$, respectively, and the sets $\check{T}_{k^{\prime}}^{+}, \check{T}_{k^{\prime}}^{-}$by

$$
\check{T}_{k^{\prime}}^{+}=\left[T_{k^{\prime}}^{+} \times \mathbb{R}\right] \cap \check{\Omega}_{\varepsilon}, \quad \check{T}_{k^{\prime}}^{-}=\left[T_{k^{\prime}}^{-} \times \mathbb{R}\right] \cap \check{\Omega}_{\varepsilon} .
$$

Note that the triangles $T_{k^{\prime}}^{+}, T_{k^{\prime}}^{-}, k^{\prime} \in \mathbb{Z}^{2}$, provide a triangulation in $\mathbb{R}^{2}$ whose vertexes are pairs of integer numbers.

For $p_{\varepsilon} \in L_{l o c}^{2}\left(\check{\Omega}_{\varepsilon}\right)$, with $\nabla p_{\varepsilon} \in H^{-1}\left(\check{\Omega}_{\varepsilon}\right)^{3}$, we define $w_{\varepsilon}$ as the unique solution of the Dirichlet problem

$$
-\Delta w_{\varepsilon}=\nabla p_{\varepsilon} \text { in } \check{\Omega}_{\varepsilon}, \quad w_{\varepsilon} \in H_{0}^{1}\left(\check{\Omega}_{\varepsilon}\right) .
$$

Observe that this problem has a unique solution because, for $\varepsilon$ small, $\check{\Omega}_{\varepsilon}$ is contained in the set $\mathbb{R}^{2} \times(-M, 1)$ for some $M>0$, and thus there exists a Poincaré constant for $\check{\Omega}_{\varepsilon}$, independent of $\varepsilon$. Moreover, the sequence $w_{\varepsilon}$ satisfies

$$
\left\|\nabla w_{\varepsilon}\right\|_{L^{2}\left(\check{\Omega}_{\varepsilon}\right)^{3}}=\left\|\nabla p_{\varepsilon}\right\|_{H^{-1}\left(\check{\Omega}_{\varepsilon}\right)^{3}} .
$$

Now, we define $p_{\varepsilon}^{0}$ as the unique continuous function in $\mathbb{R}^{2}$ such that for every $k^{\prime} \in \mathbb{Z}^{2}, p_{\varepsilon}^{0}$ is affine in the triangles $T_{k^{\prime}}^{+}, T_{k^{\prime}}^{-}$, and satisfies

$$
p_{\varepsilon}^{0}\left(k^{\prime}\right)=\int_{C_{k^{\prime}}} p_{\varepsilon}(s) d s .
$$

We also take

$$
p_{\varepsilon}^{1}=p_{\varepsilon}-p_{\varepsilon}^{0}
$$

Clearly, $p_{\varepsilon}^{0}$ belongs to $W_{\text {loc }}^{1, \infty}\left(\mathbb{R}^{2}\right) \subset H_{\text {loc }}^{1}\left(\mathbb{R}^{2}\right)$. Moreover, for every $k^{\prime} \in \mathbb{Z}^{2}$, we have

$$
\begin{aligned}
& \left\|\nabla p_{\varepsilon}^{0}\right\|_{L^{\infty}\left(T_{k}^{+}\right)^{2}}=\left(\left|p_{\varepsilon}^{0}\left(k^{\prime}+e_{1}^{\prime}\right)-p_{\varepsilon}^{0}\left(k^{\prime}\right)\right|^{2}+\left|p_{\varepsilon}^{0}\left(k^{\prime}+e_{2}^{\prime}\right)-p_{\varepsilon}^{0}\left(k^{\prime}\right)\right|^{2}\right)^{\frac{1}{2}} \\
& \quad=\left(\left|\int_{C_{k^{\prime}+e_{1}^{\prime}}} p_{\varepsilon}(s) d s-\int_{C_{k^{\prime}}} p_{\varepsilon}(s) d s\right|^{2}+\left|\int_{C_{k^{\prime}+e_{2}^{\prime}}} p_{\varepsilon}(s) d s-\int_{C_{k^{\prime}}} p_{\varepsilon}(s) d s\right|^{2}\right)^{\frac{1}{2}} .
\end{aligned}
$$

In order to estimate the two integrals on the right-hand side of (4.9), let us use Proposition 4.1 (ii) in [14] (see also [9], [17]) and definition (4.6) of $w_{\varepsilon}$, which prove that there exists $C>0$ independent of $\varepsilon$ such that

$$
\int_{\check{C}_{k^{\prime}}}\left|p_{\varepsilon}-f_{\check{C}_{k^{\prime}}} p_{\varepsilon} d s\right|^{2} d x \leq C\left\|\nabla p_{\varepsilon}\right\|_{H^{-1}\left(\check{C}_{k^{\prime}}\right)^{3}}^{2} \leq C\left\|\nabla w_{\varepsilon}\right\|_{L^{2}\left(\check{C}_{k^{\prime}}\right)^{3}}^{2} \quad \forall k^{\prime} \in \mathbb{Z}^{2} .
$$

Copyright (c) by SIAM. Unauthorized reproduction of this article is prohibited. 
Using the Cauchy-Schwarz inequality, this also implies that for every $A \subset \check{C}_{k^{\prime}}$ with $|A|>0$, we have

$$
\begin{aligned}
& \left|f_{A} p_{\varepsilon}(s) d s-f_{\check{C}_{k^{\prime}}} p_{\varepsilon}(s) d s\right| \\
& \quad \leq\left(f_{A}\left|p_{\varepsilon}(x)-f_{\check{C}_{k^{\prime}}} p_{\varepsilon}(s) d s\right|^{2} d x\right)^{1 / 2} \leq \frac{C}{|A|^{1 / 2}}\left\|\nabla w_{\varepsilon}\right\|_{L^{2}\left(\check{C}_{k^{\prime}}\right)^{3}},
\end{aligned}
$$

and then, by (4.10),

$$
\int_{\check{C}_{k^{\prime}}}\left|p_{\varepsilon}-f_{\check{A}} p_{\varepsilon} d s\right|^{2} d x \leq \frac{C}{|A|}\left\|\nabla w_{\varepsilon}\right\|_{L^{2}\left(\check{C}_{k^{\prime}}\right)^{3}}^{2} \quad \forall A \subset \check{C}_{k^{\prime}},|A|>0, \forall k^{\prime} \in \mathbb{Z}^{2}
$$

From (4.11) with $A=C_{k^{\prime}}$ and $A=C_{k^{\prime}+e_{1}^{\prime}}$, which are contained in $\check{C}_{k^{\prime}}$, we can estimate the first term on the right-hand side of (4.9) by

$$
\begin{aligned}
& \left|\int_{C_{k^{\prime}+e_{1}^{\prime}}} p_{\varepsilon}(s) d s-\int_{C_{k^{\prime}}} p_{\varepsilon}(s) d s\right| \\
& \quad \leq\left|\int_{C_{k^{\prime}+e_{1}^{\prime}}} p_{\varepsilon}(s) d s-f_{\check{C}_{k^{\prime}}} p_{\varepsilon}(s) d s\right|+\left|f_{\check{C}_{k^{\prime}}} p_{\varepsilon}(s) d s-\int_{C_{k^{\prime}}} p_{\varepsilon}(s) d s\right| \\
& \quad \leq C\left\|\nabla w_{\varepsilon}\right\|_{L^{2}\left(\check{C}_{k^{\prime}}\right)^{3}} .
\end{aligned}
$$

Using the same reasoning in the second term on the right-hand side of (4.9), we get

$$
\left\|\nabla p_{\varepsilon}^{0}\right\|_{L^{\infty}\left(T_{k^{\prime}}^{+}\right)^{2}} \leq C\left\|\nabla w_{\varepsilon}\right\|_{L^{2}\left(\check{C}_{k^{\prime}}\right)^{3}} \quad \forall k^{\prime} \in \mathbb{Z}^{2} .
$$

In the same way, we can also prove

$$
\left\|\nabla p_{\varepsilon}^{0}\right\|_{L^{\infty}\left(T_{k^{\prime}}^{-}\right)^{2}} \leq C\left\|\nabla w_{\varepsilon}\right\|_{L^{2}\left(\check{C}_{k^{\prime}}\right)^{3}} \quad \forall k^{\prime} \in \mathbb{Z}^{2} .
$$

Using that every set $\check{C}_{k^{\prime}}$ intersects at most 24 sets $\check{C}_{l^{\prime}}, l^{\prime} \neq k^{\prime}$, and (4.7), we deduce

$$
\begin{aligned}
& \int_{\mathbb{R}^{2}}\left|\nabla p_{\varepsilon}^{0}\right|^{2} d x^{\prime}=\sum_{k^{\prime} \in \mathbb{Z}^{2}}\left(\int_{T_{k^{\prime}}^{+}}\left|\nabla p_{\varepsilon}^{0}\right|^{2} d x^{\prime}+\int_{T_{k^{\prime}}^{-}}\left|\nabla p_{\varepsilon}^{0}\right|^{2} d x^{\prime}\right) \\
& \leq C \sum_{k^{\prime} \in \mathbb{Z}^{2}} \int_{\check{C}_{k^{\prime}}}\left|\nabla w_{\varepsilon}\right|^{2} d x \leq C \int_{\check{\Omega}_{\varepsilon}}\left|\nabla w_{\varepsilon}\right|^{2} d x=C\left\|\nabla p_{\varepsilon}\right\|_{H^{-1}\left(\check{\Omega}_{\varepsilon}\right)^{3}}^{2} .
\end{aligned}
$$

On the other hand, using that in each triangle $T_{k^{\prime}}^{+} p_{\varepsilon}^{0}$ is a convex combination of the values (4.8) of $p_{\varepsilon}^{0}$ on the vertexes of this triangle, and (4.12), we have for every $k^{\prime} \in \mathbb{Z}^{2}$ that

$$
\begin{aligned}
& \int_{\check{T}_{k^{\prime}}^{+}}\left|p_{\varepsilon}^{1}\right|^{2} d x=\int_{\check{T}_{k^{\prime}}^{+}}\left|p_{\varepsilon}-p_{\varepsilon}^{0}\right|^{2} d x \\
& \quad \leq \int_{\check{C}_{k^{\prime}}}\left|p_{\varepsilon}-\int_{C_{k^{\prime}}} p_{\varepsilon} d s\right|^{2} d x+\int_{\check{C}_{k^{\prime}}}\left|p_{\varepsilon}-f_{C_{k^{\prime}+e_{1}^{\prime}}} p_{\varepsilon} d s\right|^{2} d x+\int_{\check{C}_{k^{\prime}}}\left|p_{\varepsilon}-\int_{C_{k^{\prime}+e_{2}^{\prime}}} p_{\varepsilon} d s\right|^{2} d x \\
& \quad \leq C\left\|\nabla w_{\varepsilon}\right\|_{L^{2}\left(\check{C}_{k^{\prime}}\right)^{3}}^{2} .
\end{aligned}
$$

Copyright $@$ ㅇ by SIAM. Unauthorized reproduction of this article is prohibited. 
Analogously, we have

$$
\int_{\check{T}_{k^{\prime}}^{-}}\left|p_{\varepsilon}^{1}\right|^{2} d x \leq C\left\|\nabla w_{\varepsilon}\right\|_{L^{2}\left(\check{C}_{k^{\prime}}\right)^{3}}^{2}
$$

Thus, adding in $k^{\prime} \in \mathbb{Z}^{2}$, we get

$$
\left\|p_{\varepsilon}^{1}\right\|_{L^{2}\left(\check{\Omega}_{\varepsilon}\right)} \leq C\left\|\nabla p_{\varepsilon}\right\|_{H^{-1}\left(\check{\Omega}_{\varepsilon}\right)^{3}}^{2} .
$$

This inequality and (4.13) show (4.5).

Step 2. Let us now consider the case of a half-space $\omega=(0,+\infty) \times \mathbb{R}$. Once the corresponding result is proved, the general case will easily follow by using a system of local charts.

We define

$$
\Omega_{\varepsilon}^{*}=\left\{x=\left(x^{\prime}, x_{3}\right) \in \mathbb{R}^{2} \times \mathbb{R}:-\delta_{\varepsilon} \Psi\left(\frac{x^{\prime}}{r_{\varepsilon}}\right)<x_{3}<\varepsilon\right\} .
$$

For $p_{\varepsilon} \in L_{l o c}^{2}\left(\Omega_{\varepsilon}\right)$, with $\nabla p_{\varepsilon}$ in $H^{-1}\left(\Omega_{\varepsilon}\right)^{3}$, similarly as in [23], we define $p_{\varepsilon}^{*} \in L_{l o c}^{2}\left(\Omega_{\varepsilon}^{*}\right)$ by

$$
p_{\varepsilon}^{*}\left(x_{1}, x_{2}, x_{3}\right)= \begin{cases}-3 p_{\varepsilon}\left(-x_{1}, x_{2}, x_{3}\right)+4 p_{\varepsilon}\left(-2 x_{1}, x_{2}, x_{3}\right) & \text { if } x_{1}<0, \\ p_{\varepsilon}\left(x_{1}, x_{2}, x_{3}\right) & \text { if } x_{1}>0 .\end{cases}
$$

Then it is easy to check that $\nabla p_{\varepsilon}^{*}$ belongs to $H^{-1}\left(\Omega_{\varepsilon}^{*}\right)^{3}$ and

$$
\left\|\nabla p_{\varepsilon}^{*}\right\|_{H^{-1}\left(\Omega_{\varepsilon}^{*}\right)^{3}} \leq C\left\|\nabla p_{\varepsilon}\right\|_{H^{-1}\left(\Omega_{\varepsilon}\right)^{3}}
$$

with $C$ independent of $p_{\varepsilon}^{*}$. So, by Step 1 , there exist $p_{\varepsilon}^{*, 1} \in L^{2}\left(\Omega_{\varepsilon}^{*}\right)$ and $p_{\varepsilon}^{*, 0} \in H_{l o c}^{1}\left(\mathbb{R}^{2}\right)$ such that

$$
\begin{aligned}
& p_{\varepsilon}^{*}=p_{\varepsilon}^{*, 0}+p_{\varepsilon}^{*, 1} \text { in } \Omega_{\varepsilon}^{*}, \\
& \varepsilon^{\frac{3}{2}}\left\|\nabla p_{\varepsilon}^{*, 0}\right\|_{L^{2}\left(\mathbb{R}^{2}\right)^{2}}+\left\|p_{\varepsilon}^{*, 1}\right\|_{L^{2}\left(\Omega_{\varepsilon}^{*}\right)} \leq C\left\|\nabla p_{\varepsilon}^{*}\right\|_{H^{-1}\left(\Omega_{\varepsilon}^{*}\right)^{3}} \leq C\left\|\nabla p_{\varepsilon}\right\|_{H^{-1}\left(\Omega_{\varepsilon}^{*}\right)^{3} .}
\end{aligned}
$$

The restrictions $p_{\varepsilon}^{0}$ and $p_{\varepsilon}^{1}$ of $p_{\varepsilon}^{*, 0}$ and $p_{\varepsilon}^{*, 1}$ to $\Omega_{\varepsilon}$ satisfy (4.1) and (4.2).

Corollary 4.2. Let $\omega$ be a bounded domain of class $C^{2}$ of $\mathbb{R}^{2}$ and consider $\Omega_{\varepsilon}$ defined by (2.3) where $\delta_{\varepsilon}$ and $r_{\varepsilon}$ satisfy (2.1). Then there exist $\varepsilon_{0}>0$ and $C>0$, such that for every $\varepsilon \in\left(0, \varepsilon_{0}\right)$ and every $p_{\varepsilon} \in L_{0}^{2}\left(\Omega_{\varepsilon}\right)$, we have

$$
\left\|p_{\varepsilon}\right\|_{L_{0}^{2}\left(\Omega_{\varepsilon}\right)} \leq \frac{C}{\varepsilon}\left\|\nabla p_{\varepsilon}\right\|_{H^{-1}\left(\Omega_{\varepsilon}\right)^{3}} .
$$

Moreover, $p_{\varepsilon}$ can be decomposed as $p_{\varepsilon}=p_{\varepsilon}^{0}+p_{\varepsilon}^{1}$, with $p_{\varepsilon}^{0} \in H^{1}(\omega)$ (it does not depend on the variable $\left.x_{3}\right), p_{\varepsilon}^{1} \in L^{2}\left(\Omega_{\varepsilon}\right)$, and

$$
\left\|p_{\varepsilon}^{0}\right\|_{H^{1}(\omega)} \leq C \varepsilon^{-3 / 2}\left\|\nabla p_{\varepsilon}\right\|_{H^{-1}\left(\Omega_{\varepsilon}\right)^{3}}, \quad\left\|p_{\varepsilon}^{1}\right\|_{L^{2}\left(\Omega_{\varepsilon}\right)} \leq C\left\|\nabla p_{\varepsilon}\right\|_{H^{-1}\left(\Omega_{\varepsilon}\right)^{3}} .
$$

Proof. By Lemma 4.1, given $p_{\varepsilon} \in L_{0}^{2}\left(\Omega_{\varepsilon}\right)$, there exist $p_{\varepsilon}^{0} \in H_{l o c}^{1}(\omega), p_{\varepsilon}^{1} \in L^{2}\left(\Omega_{\varepsilon}\right)$ such that (4.1) and (4.2) hold, with $C$ independent of $\varepsilon$. Equality (4.1) shows in particular that $p_{\varepsilon}^{0}$ belongs to $L^{2}(\omega)$. Integrating (4.1) in $\Omega_{\varepsilon}$ and using that $p_{\varepsilon}$ has null integral, we get

$$
\int_{\Omega_{\varepsilon}} p_{\varepsilon}^{0} d x+\int_{\Omega_{\varepsilon}} p_{\varepsilon}^{1} d x=0
$$

Copyright (c) by SIAM. Unauthorized reproduction of this article is prohibited. 
and thus

$$
\begin{aligned}
& \left|f_{\omega} p_{\varepsilon}^{0} d x^{\prime}\right| \leq\left|f_{\omega} p_{\varepsilon}^{0} d x^{\prime}-f_{\Omega_{\varepsilon}} p_{\varepsilon}^{0} d x\right|+\left|f_{\Omega_{\varepsilon}} p_{\varepsilon}^{1} d x\right| \\
& =\left|f_{\Omega_{\varepsilon}}\left(p_{\varepsilon}^{0}-f_{\omega} p_{\varepsilon}^{0} d s^{\prime}\right) d x\right|+\left|f_{\Omega_{\varepsilon}} p_{\varepsilon}^{1} d x\right| \leq C|| p_{\varepsilon}^{0}-f_{\omega} p_{\varepsilon}^{0} d s^{\prime}\left\|_{L^{2}(\omega)}+\frac{1}{\left|\Omega_{\varepsilon}\right|^{\frac{1}{2}}}\right\| p_{\varepsilon}^{1} \|_{L^{2}\left(\Omega_{\varepsilon}\right)} .
\end{aligned}
$$

The first term on the right-hand side of this inequality can be estimated by using the Poincaré-Wirtinger inequality, which gives

$$
\left\|p_{\varepsilon}^{0}-f_{\omega} p_{\varepsilon}^{0} d s^{\prime}\right\|_{L^{2}(\omega)} \leq C\left\|\nabla p_{\varepsilon}^{0}\right\|_{L^{2}(\omega)^{2}}
$$

and therefore by (4.2) we get

$$
\left|f_{\omega} p_{\varepsilon}^{0} d x^{\prime}\right| \leq C \varepsilon^{-3 / 2}\left\|\nabla p_{\varepsilon}\right\|_{H^{-1}\left(\Omega_{\varepsilon}\right)^{3}}+C \varepsilon^{-1 / 2}\left\|\nabla p_{\varepsilon}\right\|_{H^{-1}\left(\Omega_{\varepsilon}\right)^{3}} \leq C \varepsilon^{-3 / 2}\left\|\nabla p_{\varepsilon}\right\|_{H^{-1}\left(\Omega_{\varepsilon}\right)^{3}} .
$$

Taking into account this estimate in (4.16) and using again (4.2), we have then proved

$$
\left\|p_{\varepsilon}^{0}\right\|_{L^{2}(\omega)} \leq C \varepsilon^{-3 / 2}\left\|\nabla p_{\varepsilon}\right\|_{H^{-1}\left(\Omega_{\varepsilon}\right)^{3}}
$$

which, combined with (4.2), proves (4.15).

To finish the proof it is enough to remark that (4.15) implies

$$
\left\|p_{\varepsilon}\right\|_{L^{2}\left(\Omega_{\varepsilon}\right)} \leq C \varepsilon^{1 / 2}\left\|p_{\varepsilon}^{0}\right\|_{L^{2}(\omega)}+\left\|p_{\varepsilon}^{1}\right\|_{L^{2}\left(\Omega_{\varepsilon}\right)} \leq C \varepsilon^{-1}\left\|\nabla p_{\varepsilon}\right\|_{H^{-1}\left(\Omega_{\varepsilon}\right)^{3}} .
$$

Corollary 4.3. Let $\omega$ be a bounded domain of class $C^{2}$ of $\mathbb{R}^{2}$ and consider $\Omega_{\varepsilon}$ defined by (2.3) where $\delta_{\varepsilon}$ and $r_{\varepsilon}$ satisfy (2.1). Then there exist $\varepsilon_{0}>0$ and $C>0$, such that for every $\varepsilon \in\left(0, \varepsilon_{0}\right)$ and every $p_{\varepsilon} \in L_{0}^{2}\left(\Omega_{\varepsilon}\right)$ there exists $v_{\varepsilon} \in H_{0}^{1}\left(\Omega_{\varepsilon}\right)^{3}$ satisfying $\operatorname{div} v_{\varepsilon}=p_{\varepsilon}$ in $\Omega_{\varepsilon}$ and

$$
\left\|v_{\varepsilon}\right\|_{H_{0}^{1}\left(\Omega_{\varepsilon}\right)^{3}} \leq \frac{C}{\varepsilon}\left\|p_{\varepsilon}\right\|_{L^{2}\left(\Omega_{\varepsilon}\right)} .
$$

Proof. Since $\Omega_{\varepsilon}$ is Lipschitz, it is well known that (using that $\nabla: L_{0}^{2}\left(\Omega_{\varepsilon}\right) \rightarrow$ $H^{-1}\left(\Omega_{\varepsilon}\right)^{3}$ has closed range), given $p_{\varepsilon} \in L_{0}^{2}\left(\Omega_{\varepsilon}\right)$, there exists $v_{\varepsilon}^{*} \in H_{0}^{1}\left(\Omega_{\varepsilon}\right)^{3}$ with $\operatorname{div} v_{\varepsilon}^{*}=p_{\varepsilon}$ in $\Omega_{\varepsilon}$. This proves the existence of the $\left(v_{\varepsilon}, q_{\varepsilon}\right) \in H_{0}^{1}\left(\Omega_{\varepsilon}\right)^{3} \times L_{0}^{2}\left(\Omega_{\varepsilon}\right)$ solution of the Stokes problem

$$
\left\{\begin{array}{l}
-\Delta v_{\varepsilon}+\nabla q_{\varepsilon}=0 \text { in } \Omega_{\varepsilon}, \\
\operatorname{div} v_{\varepsilon}=p_{\varepsilon} \text { in } \Omega_{\varepsilon} \\
v_{\varepsilon}=0 \text { on } \partial \Omega_{\varepsilon} .
\end{array}\right.
$$

Showing that $v_{\varepsilon}$ satisfies (4.17), we then get the result. For this purpose, we use $v_{\varepsilon}$ as a test function in (4.18), which, applying (4.14) to $q_{\varepsilon}$, gives

$$
\begin{aligned}
& \left\|D v_{\varepsilon}\right\|_{L^{2}\left(\Omega_{\varepsilon}\right)^{3 \times 3}}^{2}=\int_{\Omega_{\varepsilon}} q_{\varepsilon} \operatorname{div} v_{\varepsilon} d x=\int_{\Omega_{\varepsilon}} q_{\varepsilon} p_{\varepsilon} d x \leq C \varepsilon^{-1}\left\|\nabla q_{\varepsilon}\right\|_{H^{-1}\left(\Omega_{\varepsilon}\right)^{3}}\left\|p_{\varepsilon}\right\|_{L^{2}\left(\Omega_{\varepsilon}\right)} \\
& =C \varepsilon^{-1}\left\|\Delta v_{\varepsilon}\right\|_{H^{-1}\left(\Omega_{\varepsilon}\right)^{3}}\left\|p_{\varepsilon}\right\|_{L^{2}\left(\Omega_{\varepsilon}\right)} \leq C \varepsilon^{-1}\left\|D v_{\varepsilon}\right\|_{L^{2}\left(\Omega_{\varepsilon}\right)^{3 \times 3}}\left\|p_{\varepsilon}\right\|_{L^{2}\left(\Omega_{\varepsilon}\right)}
\end{aligned}
$$

and then (4.17).

Copyright (c) by SIAM. Unauthorized reproduction of this article is prohibited. 
Proposition 4.4. Let $\omega$ be a bounded domain of class $C^{2}$ of $\mathbb{R}^{2}$ and consider $\Omega_{\varepsilon}$ defined by (2.3) where $\delta_{\varepsilon}$ and $r_{\varepsilon}$ satisfy (2.1). Then there exist $\varepsilon_{0}>0$ and $C>0$, such that for every $\varepsilon \in\left(0, \varepsilon_{0}\right)$ and every $w_{\varepsilon} \in H^{1}\left(\Omega_{\varepsilon}\right)$ with $w_{\varepsilon}=0$ on $\omega \times\{\varepsilon\}$, we have the following:

$$
\left\|w_{\varepsilon}\right\|_{L^{2}\left(\Omega_{\varepsilon}\right)} \leq C \varepsilon\left\|\partial_{x_{3}} w_{\varepsilon}\right\|_{L^{2}\left(\Omega_{\varepsilon}\right)} .
$$

(ii) The function $w_{\varepsilon}$ belongs to $L^{6}\left(\Omega_{\varepsilon}\right)$ and

$$
\left\|w_{\varepsilon}\right\|_{L^{6}\left(\Omega_{\varepsilon}\right)} \leq C\left\|\nabla w_{\varepsilon}\right\|_{L^{2}\left(\Omega_{\varepsilon}\right)^{3}} .
$$

Proof. Statement (i) easily follows using that

$$
w_{\varepsilon}(x)=-\int_{x_{3}}^{\varepsilon} \partial_{x_{3}} w_{\varepsilon}\left(x^{\prime}, t\right) d t, \text { a.e. } x \in \Omega_{\varepsilon} .
$$

In order to prove (4.20), we extend $w_{\varepsilon}(x)$ by zero for $x_{3}>\varepsilon$. Then $w_{\varepsilon}$ belongs to $H^{1}\left(\Omega_{\varepsilon}^{*}\right)$, with

$$
\Omega_{\varepsilon}^{*}=\left\{x \in \mathbb{R}^{3}: x^{\prime} \in \omega,-\delta_{\varepsilon} \Psi\left(\frac{x^{\prime}}{r_{\varepsilon}}\right)<x_{3}<1\right\},
$$

and thus the result follows from Proposition 4.1 (iii) in [14].

Proof of Theorem 3.1. Taking into account that $\Omega_{\varepsilon}$ is Lipschitz and then that $C^{1}(\bar{\Omega})^{3}$ is dense in $H^{1}(\Omega)^{3}$ and that Sobolev's inequality holds, we have that every function $v \in H^{1}\left(\Omega_{\varepsilon}\right)^{3}$ satisfies

$$
\int_{\Omega_{\varepsilon}}(v \cdot \nabla) v v d x=\frac{1}{2} \int_{\Omega_{\varepsilon}} v \nabla|v|^{2} d x=\int_{\partial \Omega_{\varepsilon}}|v|^{2} v \nu d \sigma-\int_{\Omega_{\varepsilon}}|v|^{2} \operatorname{div} v d x
$$

and so, if $v$ also satisfies $v \nu=0$ on $\partial \Omega_{\varepsilon}$, $\operatorname{div} v=0$ in $\Omega_{\varepsilon}$, we get that

$$
\int_{\Omega_{\varepsilon}}(v \cdot \nabla) v v d x=0
$$

This allows us to repeat the classical proof of the existence of solution for the NavierStokes problem with homogeneous Dirichlet conditions (see, e.g., [21, Theorem 7.1, Chapter 1], [24, Theorem 10.1]) to obtain the existence of solution for problem (3.1). Using $u_{\varepsilon}$ as a test function in (3.1) and taking into account that $\operatorname{div} u_{\varepsilon}=0$ in $\Omega_{\varepsilon}$ and the boundary conditions imposed to $u_{\varepsilon}$, we have

$$
\mu \int_{\Omega_{\varepsilon}}\left|D u_{\varepsilon}\right|^{2} d x+\frac{\gamma}{\varepsilon} \int_{\Gamma_{\varepsilon}}\left|u_{\varepsilon}\right|^{2} d \sigma=\int_{\Omega_{\varepsilon}} f_{\varepsilon} u_{\varepsilon} d x
$$

where, thanks to the structure (3.2) of $f_{\varepsilon}$ and estimate (4.19) applied to $u_{\varepsilon}$, we can estimate the right-hand side by

$$
\int_{\Omega_{\varepsilon}} f_{\varepsilon} u_{\varepsilon} d x \leq C \varepsilon^{\frac{3}{2}}\left\|D u_{\varepsilon}\right\|_{L^{2}\left(\Omega_{\varepsilon}\right)^{3 \times 3}} .
$$

Thus, substituting in (4.21), we deduce the second estimate in (3.3) and then by (4.19) the first one. 
Equation

$$
-\mu \Delta u_{\varepsilon}+\nabla p_{\varepsilon}+\left(u_{\varepsilon} \cdot \nabla\right) u_{\varepsilon}=f_{\varepsilon} \text { in } \Omega_{\varepsilon}
$$

combined with the first and second assertions in (3.3) and $\left\|u_{\varepsilon}\right\|_{L^{6}\left(\Omega_{\varepsilon}\right)^{3}} \leq C \varepsilon^{\frac{3}{2}}$ (using (4.20) and (3.3)) now proves that $\left\|\nabla p_{\varepsilon}\right\|_{H^{-1}\left(\Omega_{\varepsilon}\right)^{3}} \leq C \varepsilon^{\frac{3}{2}}$. Then, by Corollary 4.2, we get the last estimate in (3.3) and that $p_{\varepsilon}$ can be decomposed as $p_{\varepsilon}=p_{\varepsilon}^{0}+p_{\varepsilon}^{1}$, with $p_{\varepsilon}^{0}$ in $H^{1}(\omega)$ and $p_{\varepsilon}^{1}$ in $L^{2}\left(\Omega_{\varepsilon}\right)$ satisfying $(3.4)$.

5. Some compactness results. In this section we obtain some compactness results about the behavior of a sequence $\left(u_{\varepsilon}, p_{\varepsilon}\right)$ satisfying the a priori estimates (3.3) and (3.4) combined with the boundary conditions $u_{\varepsilon}=0$ on $\omega \times\{\varepsilon\}, u_{\varepsilon} \nu=0$ on $\partial \Omega_{\varepsilon} \backslash(\omega \times\{\varepsilon\})$, but where $\left(u_{\varepsilon}, p_{\varepsilon}\right)$ is not necessarily the solution of any PDE.

LEMMA 5.1. Let $u_{\varepsilon}$ be in $H^{1}\left(\Omega_{\varepsilon}\right)^{3}$ with $u_{\varepsilon}=0$ on $\omega \times\{\varepsilon\}, u_{\varepsilon} \nu=0$ on $\partial \Omega_{\varepsilon} \backslash$ $(\omega \times\{\varepsilon\})$, and such that there exists a constant $C$ independent of $\varepsilon$ satisfying

$$
\begin{gathered}
f_{\Omega_{\varepsilon}}\left|D u_{\varepsilon}\right|^{2} d x \leq C \varepsilon^{2}, \\
f_{\Omega_{\varepsilon}}\left|\operatorname{div} u_{\varepsilon}\right|^{2} d x \leq C \varepsilon^{4} .
\end{gathered}
$$

Let us define $\tilde{u}_{\varepsilon} \in H^{1}\left(\tilde{\Omega}_{\varepsilon}\right)^{3}$ by (3.6). Then, for a subsequence of $\varepsilon$ still denoted by $\varepsilon$, there exist $\tilde{u}^{\prime} \in H^{1}\left(0,1 ; L^{2}(\omega)\right)^{2}, \tilde{w} \in H^{1}\left(0,1 ; H^{-1}(\omega)\right)$, and $\tilde{\pi} \in L^{2}(\Omega)$ such that

$$
\begin{gathered}
\tilde{u}^{\prime}(1)=0 \text { in } L^{2}(\omega), \quad \tilde{w}(0)=\tilde{w}(1)=0 \text { in } H^{-1}(\omega), \\
\operatorname{div}_{y^{\prime}} \tilde{u}^{\prime}+\partial_{y_{3}} \tilde{w}=\tilde{\pi} \text { in } H^{1}\left(0,1 ; H^{-1}(\omega)\right), \\
\operatorname{div}_{y^{\prime}} \int_{0}^{1} \tilde{u}^{\prime}\left(y^{\prime}, t\right) d t=\int_{0}^{1} \tilde{\pi}\left(y^{\prime}, t\right) d t \text { in } L^{2}(\omega), \\
\int_{0}^{1} \tilde{u}^{\prime}\left(y^{\prime}, t\right) d t \nu=0 \text { in } H^{-\frac{1}{2}}(\partial \omega), \\
\frac{\tilde{u}_{\varepsilon}}{\varepsilon} \rightarrow 0 \text { in } H^{1}(\Omega)^{3} \\
\frac{\tilde{u}_{\varepsilon}}{\varepsilon^{2}} \rightarrow\left(\tilde{u}^{\prime}, 0\right) \text { in } H^{1}\left(0,1 ; L^{2}(\omega)\right)^{3} \\
\frac{1}{\varepsilon^{3}} \operatorname{div}_{y^{\prime}} \tilde{u}_{\varepsilon}^{\prime}+\frac{1}{\varepsilon^{3}} \partial_{y_{3}} \tilde{u}_{\varepsilon, 3} \rightarrow \tilde{\pi} \text { in } H^{1}\left(0,1 ; H^{-1}(\omega)\right)
\end{gathered}
$$

Moreover, if $\operatorname{div} u_{\varepsilon}=0$ in $\Omega_{\varepsilon}$, then $\tilde{\pi}=0, \tilde{w}$ is in $H^{2}\left(0,1 ; H^{-1}(\omega)\right)$, and convergence (5.9) holds in $H^{2}\left(0,1 ; H^{-1}(\omega)\right)$.

Proof. Since $u_{\varepsilon}$ vanishes on $\omega \times\{\varepsilon\}$, estimates (4.19) and (5.1) imply that $u_{\varepsilon}$ also satisfies

$$
\frac{1}{\varepsilon} \int_{\Omega_{\varepsilon}}\left|u_{\varepsilon}\right|^{2} d x \leq C \varepsilon^{4} .
$$

Copyright (C) by SIAM. Unauthorized reproduction of this article is prohibited. 
This inequality combined with the change of variables (3.5) and inequalities (5.1) and (5.2) imply that $\tilde{u}_{\varepsilon}$ satisfies

$$
\begin{gathered}
\int_{\tilde{\Omega}_{\varepsilon}}\left|\tilde{u}_{\varepsilon}\right|^{2} d y \leq C \varepsilon^{4}, \quad \int_{\tilde{\Omega}_{\varepsilon}}\left(\left|\nabla_{y^{\prime}} \tilde{u}_{\varepsilon}\right|^{2}+\frac{1}{\varepsilon^{2}}\left|\partial_{y_{3}} \tilde{u}_{\varepsilon}\right|^{2}\right) d y \leq C \varepsilon^{2}, \\
\int_{\tilde{\Omega}_{\varepsilon}}\left|\operatorname{div}_{y^{\prime}} \tilde{u}_{\varepsilon}^{\prime}+\frac{1}{\varepsilon} \partial_{y_{3}} \tilde{u}_{\varepsilon, 3}\right|^{2} d y \leq C \varepsilon^{4} .
\end{gathered}
$$

Therefore, up to a subsequence, there exist $\tilde{u} \in H^{1}\left(0,1 ; L^{2}(\omega)\right)^{3}$, with $\tilde{u}(1)=0$, and $\tilde{\pi} \in L^{2}(\Omega)$ such that

$$
\frac{\tilde{u}_{\varepsilon}}{\varepsilon^{2}} \rightarrow \tilde{u} \text { in } H^{1}\left(0,1 ; L^{2}(\omega)\right)^{3},
$$

and (5.8), (5.10) hold. By (5.13), we also have that

$$
\frac{1}{\varepsilon^{2}} \operatorname{div}_{y^{\prime}} \tilde{u}_{\varepsilon}^{\prime} \rightarrow \operatorname{div}_{y^{\prime}} \tilde{u}^{\prime} \quad \text { in } H^{1}\left(0,1 ; H^{-1}(\omega)\right),
$$

and then (5.10) implies that $\partial_{y_{3}} \tilde{u}_{\varepsilon, 3} / \varepsilon^{3}$ is bounded in $L^{2}\left(0,1 ; H^{-1}(\omega)\right)$. Using then that $\tilde{u}_{\varepsilon, 3}=0$ on $\omega \times\{1\}$, we deduce that $\tilde{u}_{\varepsilon, 3} / \varepsilon^{3}$ is bounded in $H^{1}\left(0,1 ; H^{-1}(\omega)\right)$, and therefore, up to a subsequence, there exists $\tilde{w} \in H^{1}\left(0,1 ; H^{-1}(\omega)\right)$, with $\tilde{w}(1)=0$ in $H^{-1}(\omega)$, such that (5.9) holds. By (5.13), we get that $\tilde{u}_{3}=0$, which finishes the proof of (5.8). From (5.9), (5.10), and (5.14), we also deduce (5.4).

Now, we consider $\eta \in C^{\infty}(\omega)$. Integrating by parts into $\tilde{\Omega}_{\varepsilon}$ and taking into account that $u_{\varepsilon} \nu=0$ on $\partial \Omega_{\varepsilon}$, we get

$$
\int_{\widetilde{\Omega}_{\varepsilon}}\left(\frac{1}{\varepsilon^{2}} \operatorname{div}_{y^{\prime}} \tilde{u}_{\varepsilon}^{\prime}+\frac{1}{\varepsilon^{3}} \partial_{y_{3}} \tilde{u}_{\varepsilon, 3}\right) \eta\left(y^{\prime}\right) d y=-\int_{\widetilde{\Omega}_{\varepsilon}} \frac{\tilde{u}_{\varepsilon}^{\prime}(y)}{\varepsilon^{2}} \nabla_{y^{\prime}} \eta\left(y^{\prime}\right) d y .
$$

Since (5.11) and (5.12) imply

$$
\int_{\widetilde{\Omega}_{\varepsilon} \backslash \Omega}\left|\frac{\tilde{u}_{\varepsilon}^{\prime}}{\varepsilon^{2}}\right| d y \rightarrow 0, \quad \int_{\widetilde{\Omega}_{\varepsilon} \backslash \Omega}\left|\frac{1}{\varepsilon^{2}} \operatorname{div}_{y^{\prime}}\left(\tilde{u}_{\varepsilon}^{\prime}\right)+\frac{1}{\varepsilon^{3}} \partial_{y_{3}} \tilde{u}_{\varepsilon, 3}\right| d y \rightarrow 0,
$$

we can write the previous equality as

$$
\int_{\Omega}\left(\frac{1}{\varepsilon^{2}} \operatorname{div}_{y^{\prime}} \tilde{u}_{\varepsilon}^{\prime}+\frac{1}{\varepsilon^{3}} \partial_{y_{3}} \tilde{u}_{\varepsilon, 3}\right) \eta\left(y^{\prime}\right) d y=-\int_{\Omega} \frac{\tilde{u}_{\varepsilon}^{\prime}(y)}{\varepsilon^{2}} \nabla_{y^{\prime}} \eta\left(y^{\prime}\right) d y+O_{\varepsilon} .
$$

Passing to the limit in this equality by means of (5.8) and (5.10), we get

$$
\int_{\omega} \int_{0}^{1} \tilde{\pi}\left(y^{\prime}, y_{3}\right) d y_{3} \eta\left(y^{\prime}\right) d y^{\prime}=-\int_{\omega} \int_{0}^{1} \tilde{u}^{\prime}\left(y^{\prime}, y_{3}\right) d y_{3} \nabla_{y^{\prime}} \eta\left(y^{\prime}\right) d y^{\prime},
$$

which implies (5.5) and (5.6). Integrating (5.4) into $(0,1)$, we now deduce that $\tilde{w}(0)=$ 0 , which concludes the proof of (5.3).

Finally, if we assume that $\operatorname{div} u_{\varepsilon}=0$ in $\Omega_{\varepsilon}$, we have

$$
\frac{1}{\varepsilon^{2}} \operatorname{div}_{y^{\prime}} \tilde{u}_{\varepsilon}^{\prime}+\frac{1}{\varepsilon^{3}} \partial_{y_{3}} \tilde{u}_{\varepsilon, 3}=0 \text { in } L^{2}\left(\tilde{\Omega}_{\varepsilon}\right)
$$

which combined with (5.10) proves that $\tilde{\pi}$ is the null function. Moreover (5.14) and (5.15) imply that $\partial_{y_{3}} \tilde{u}_{\varepsilon, 3} / \varepsilon^{3}$ is bounded in $H^{1}\left(0,1 ; H^{-1}(\omega)\right)$. Therefore convergence (5.9) holds in fact in $H^{2}\left(0,1 ; H^{-1}(\omega)\right)$, and so $\tilde{w}$ is in $H^{2}\left(0,1 ; H^{-1}(\omega)\right)$.

Copyright $@$ by SIAM. Unauthorized reproduction of this article is prohibited. 
The change of variables (3.5) does not provide the information we need about the behavior of $u_{\varepsilon}$ in the part of $\Omega_{\varepsilon}$ close to $\Gamma_{\varepsilon}$. To solve this difficulty, we introduce an adaptation of the unfolding method (see [4], [11], [13], [14], [18], and [20]), which is strongly related to the two-scale convergence method (see [1], [22]). For this purpose, given $u_{\varepsilon} \in H^{1}\left(\Omega_{\varepsilon}\right)^{3}, u_{\varepsilon}=0$ on $\partial \Omega_{\varepsilon} \backslash \Gamma_{\varepsilon}$, and assuming $u_{\varepsilon}$ extended by zero to the set $\Lambda_{\varepsilon}$ given by $(2.2)$, we define $\widehat{u}_{\varepsilon}$ by

$$
\widehat{u}_{\varepsilon}\left(x^{\prime}, z\right)=u_{\varepsilon}\left(r_{\varepsilon} \kappa\left(\frac{x^{\prime}}{r_{\varepsilon}}\right)+r_{\varepsilon} z^{\prime}, r_{\varepsilon} z_{3}\right) \text {, a.e. }\left(x^{\prime}, z^{\prime}\right) \in \mathbb{R}^{2} \times \widehat{Z}_{\varepsilon},
$$

with

$$
\widehat{Z}_{\varepsilon}=\left\{z \in Z^{\prime} \times \mathbb{R}:-\frac{\delta_{\varepsilon}}{r_{\varepsilon}} \Psi\left(z^{\prime}\right)<z_{3}<\frac{\varepsilon}{r_{\varepsilon}}\right\}
$$

Remark 5.2. For $k^{\prime} \in \mathbb{Z}^{2}$ the restriction of $\widehat{u}_{\varepsilon}$ to $C_{r_{\varepsilon}}^{k^{\prime}} \times \widehat{Z}_{\varepsilon}$ does not depend on $x^{\prime}$, whereas as a function of $z$ it is obtained from $u_{\varepsilon}$ by using the change of variables

$$
z^{\prime}=\frac{x^{\prime}-r_{\varepsilon} k^{\prime}}{r_{\varepsilon}}, \quad z_{3}=\frac{x_{3}}{r_{\varepsilon}},
$$

which transforms $Q_{r_{\varepsilon}}^{k^{\prime}}$ into $\widehat{Z}_{\varepsilon}$. Therefore, the idea in the definition of the function $\hat{u}_{\varepsilon}$ is to realize a dilatation in order to study the behavior of $u_{\varepsilon}$ at a very small distance of $\Gamma_{\varepsilon}$. In addition, we observe that the change of variables (5.17), with $x^{\prime}$ fixed, transforms $\Gamma_{\varepsilon}$ into the surface $z_{3}=-\delta_{\varepsilon} / r_{\varepsilon} \Psi\left(z^{\prime}\right)$, which, thanks to the assumption $\delta_{\varepsilon} / r_{\varepsilon}$ converging to zero, almost agrees with the plane boundary $z_{3}=0$.

We will use the following lemma, whose proof is elementary and thus omitted.

Lemma 5.3. Let $v_{\varepsilon} \in L^{2}\left(\mathbb{R}^{2}\right)$ be a sequence which converges weakly in $L^{2}\left(\mathbb{R}^{2}\right)$ to a function $v$. We define $\bar{v}_{\varepsilon} \in L^{2}\left(\mathbb{R}^{2}\right)$ by

$$
\bar{v}_{\varepsilon}\left(x^{\prime}\right)=f_{C_{r_{\varepsilon}}\left(x^{\prime}\right)} v_{\varepsilon}\left(\eta^{\prime}\right) d \eta^{\prime}, \quad \text { a.e. } x^{\prime} \in \mathbb{R}^{2} .
$$

Then we have the following:

(i) The sequence $\bar{v}_{\varepsilon}$ converges weakly to $v$ in $L^{2}\left(\mathbb{R}^{2}\right)$. Moreover, if the convergence of $v_{\varepsilon}$ is strong in $L^{2}\left(\mathbb{R}^{2}\right)$, then the convergence of $\bar{v}_{\varepsilon}$ is also strong in $L^{2}\left(\mathbb{R}^{2}\right)$.

(ii) For every $\tau^{\prime} \in \mathbb{R}^{2}$, we have

$$
\frac{\bar{v}_{\varepsilon}\left(x^{\prime}+r_{\varepsilon} \tau^{\prime}\right)-\bar{v}_{\varepsilon}\left(x^{\prime}\right)}{r_{\varepsilon}} \rightarrow \nabla v \tau^{\prime} \text { in } H^{-1}\left(\mathbb{R}^{2}\right)
$$

LEMMA 5.4. We consider a sequence $u_{\varepsilon} \in H^{1}\left(\Omega_{\varepsilon}\right)^{3}$ satisfying (5.1), $u_{\varepsilon}=0$ on $\omega \times\{\varepsilon\}, u_{\varepsilon} \nu=0$ on $\partial \Omega_{\varepsilon} \backslash(\omega \times\{\varepsilon\})$. We define $\tilde{u}_{\varepsilon} \in H^{1}\left(\widetilde{\Omega}_{\varepsilon}\right)^{3}$ by (3.6) and suppose there exists $\tilde{u}^{\prime} \in H^{1}\left(0,1 ; L^{2}(\omega)\right)^{2}$ such that (5.8) holds. We also assume that there exists the limit $\lambda$ given by (3.7) and that $\lambda$ belongs to $(0,+\infty]$. Then we have the following:

(i) If $\lambda=+\infty$, then

$$
\tilde{u}^{\prime}\left(x^{\prime}, 0\right) \nabla \Psi\left(z^{\prime}\right)=0, \quad \text { a.e. }\left(x^{\prime}, z^{\prime}\right) \in \omega \times Z^{\prime} .
$$

Copyright (c) by SIAM. Unauthorized reproduction of this article is prohibited. 
(ii) If $\lambda \in(0,+\infty)$, then there exists $\widehat{u} \in L^{2}\left(\omega ; \mathcal{V}^{3}\right)$ with

$$
\widehat{u}_{3}\left(x^{\prime}, z^{\prime}, 0\right)=-\lambda \nabla \Psi\left(z^{\prime}\right) \tilde{u}^{\prime}\left(x^{\prime}, 0\right), \quad \text { a.e. }\left(x^{\prime}, z^{\prime}\right) \in \omega \times Z^{\prime},
$$

such that for every $M>0$, the sequence $\widehat{u}_{\varepsilon}$ defined by (5.16) satisfies

$$
\frac{1}{\varepsilon^{\frac{3}{2}} r_{\varepsilon}^{\frac{1}{2}}} D_{z} \widehat{u}_{\varepsilon} \rightarrow D_{z} \widehat{u} \quad \text { in } L^{2}\left(\omega \times \widehat{Q}_{M}\right)^{3 \times 3} \text {. }
$$

In addition, if $\operatorname{div} u_{\varepsilon}=0$ in $\Omega_{\varepsilon}$, then

$$
\operatorname{div}_{z} \widehat{u}=0 \quad \text { in } \omega \times \widehat{Q} .
$$

Proof. We proceed in four steps.

Step 1. Let us obtain some estimates for the sequence $\widehat{u}_{\varepsilon}$ defined by (5.16).

For $M>0$, definition (5.16) of $\widehat{u}_{\varepsilon}$ and (5.1) prove that for every $\varepsilon>0$ small enough (depending on $M$ ), we have

$$
\begin{aligned}
\int_{\mathbb{R}^{2} \times \widehat{Q}_{M}}\left|D_{z} \widehat{u}_{\varepsilon}\right|^{2} d x^{\prime} d z & =r_{\varepsilon}^{4} \sum_{k^{\prime} \in \mathbb{Z}^{2}} \int_{\widehat{Q}_{M}}\left|D u_{\varepsilon}\left(r_{\varepsilon}\left(k^{\prime}+z^{\prime}\right), r_{\varepsilon} z_{3}\right)\right|^{2} d z \\
& \leq \sum_{k^{\prime} \in \mathbb{Z}^{2}} r_{\varepsilon} \int_{Q_{r_{\varepsilon}}^{k^{\prime}}}\left|D u_{\varepsilon}\right|^{2} d x \leq r_{\varepsilon} \int_{\Omega_{\varepsilon}}\left|D u_{\varepsilon}\right|^{2} d x \leq C r_{\varepsilon} \varepsilon^{3} .
\end{aligned}
$$

On the other hand, defining

$$
\bar{u}_{\varepsilon}\left(x^{\prime}\right)=f_{C_{r_{\varepsilon}}\left(x^{\prime}\right)} u_{\varepsilon}\left(\tau^{\prime}, 0\right) d \tau=f_{C_{r_{\varepsilon}}\left(x^{\prime}\right)} \tilde{u}_{\varepsilon}\left(\tau^{\prime}, 0\right) d \tau=\int_{Z^{\prime}} \widehat{u}_{\varepsilon}\left(x^{\prime}, z^{\prime}, 0\right) d z^{\prime},
$$

a.e. $x^{\prime} \in \mathbb{R}^{2}$, using the inequality

$$
\int_{\omega} \int_{\widehat{Q}_{M}}\left|\widehat{u}_{\varepsilon}\left(x^{\prime}, z\right)-\bar{u}_{\varepsilon}\left(x^{\prime}\right)\right|^{2} d z \leq C\left(1+M^{2}\right) \int_{\mathbb{R}^{2}} \int_{\widehat{Q}_{M}}\left|D_{z} \widehat{u}_{\varepsilon}\right|^{2} d z d x^{\prime},
$$

and taking into account (5.22), we deduce that

$$
\widehat{U}_{\varepsilon}=\frac{\widehat{u}_{\varepsilon}\left(x^{\prime}, z\right)-\bar{u}_{\varepsilon}}{\varepsilon^{\frac{3}{2}} r_{\varepsilon}^{\frac{1}{2}}} \quad \text { is bounded in } L^{2}\left(\omega ; H^{1}\left(\widehat{Q}_{M}\right)^{3}\right) \quad \forall M>0 .
$$

Thus, there exists $\widehat{u} \in L^{2}\left(\omega ; H^{1}\left(\widehat{Q}_{M}\right)^{3}\right)$ for every $M>0$, such that, up to a subsequence,

$$
\widehat{U}_{\varepsilon} \rightarrow \widehat{u} \text { in } L^{2}\left(\omega ; H^{1}\left(\widehat{Q}_{M}\right)^{3}\right) \quad \forall M>0
$$

and then

$$
\frac{1}{\varepsilon^{\frac{3}{2}} r_{\varepsilon}^{\frac{1}{2}}} D_{z} \widehat{u}_{\varepsilon} \rightarrow D_{z} \widehat{u} \text { in } L^{2}\left(\omega \times \widehat{Q}_{M}\right)^{3 \times 3} \quad \forall M>0 .
$$

By semicontinuity, inequality (5.22) proves

$$
\int_{\omega \times \widehat{Q}_{M}}\left|D_{z} \widehat{u}\right|^{2} d x^{\prime} d z \leq C \quad \forall M>0 .
$$

Copyright $@$ by SIAM. Unauthorized reproduction of this article is prohibited. 
Once we prove the $Z^{\prime}$-periodicity of $\widehat{u}$ in $z^{\prime}$ (Step 2), the arbitrariness of $M$ will then imply that $\widehat{u}$ belongs to $L^{2}\left(\omega ; \mathcal{V}^{3}\right)$.

If we assume that $\operatorname{div} u_{\varepsilon}=0$ in $\Omega_{\varepsilon}$, then by definition (5.16) of $\widehat{u}_{\varepsilon}$, we have $\operatorname{div}_{z} \widehat{u}_{\varepsilon}=0$ in $\mathbb{R}^{2} \times \widehat{Q}_{M}$, which combined with (5.27) proves (5.21).

Step 2. Let us prove that $\widehat{u}$ is $Z^{\prime}$-periodic in the variable $z^{\prime}$.

We observe that by definition (5.16) of $\widehat{u}_{\varepsilon}$, for every $M>0$, we have

$$
\widehat{u}_{\varepsilon}\left(x_{1}+r_{\varepsilon}, x_{2},-\frac{1}{2}, z_{2}, z_{3}\right)=\widehat{u}_{\varepsilon}\left(x_{1}, x_{2}, \frac{1}{2}, z_{2}, z_{3}\right),
$$

a.e. $\left(x^{\prime}, z_{2}, z_{3}\right) \in \mathbb{R}^{2} \times\left(-\frac{1}{2}, \frac{1}{2}\right) \times(0, M)$, which implies

$$
\widehat{U}_{\varepsilon}\left(x_{1}+r_{\varepsilon}, x_{2},-\frac{1}{2}, z_{2}, z_{3}\right)-\widehat{U}_{\varepsilon}\left(x_{1}, x_{2}, \frac{1}{2}, z_{2}, z_{3}\right)=-\frac{\bar{u}_{\varepsilon}\left(x_{1}+r_{\varepsilon}, x_{2}\right)-\bar{u}_{\varepsilon}\left(x^{\prime}\right)}{\varepsilon^{\frac{3}{2}} r_{\varepsilon}^{\frac{1}{2}}} .
$$

Since $u_{\varepsilon}\left(x^{\prime}, 0\right) / \varepsilon^{2}$ is bounded in $L^{2}\left(\mathbb{R}^{2}\right)^{3}$, we can apply Lemma 5.3 (ii) to deduce that the right-hand side of the above equality tends to zero in $H^{-1}\left(\mathbb{R}^{2}\right)$. Therefore, passing to the limit in the previous equation by (5.26) and taking into account the arbitrariness of $M$, we get

$$
\widehat{u}\left(x^{\prime},-\frac{1}{2}, z_{2}, z_{3}\right)-\widehat{u}\left(x^{\prime}, \frac{1}{2}, z_{2}, z_{3}\right)=0 \quad \text { a.e. }\left(x^{\prime}, z_{2}, z_{3}\right) \in \omega \times\left(\frac{-1}{2}, \frac{1}{2}\right) \times \mathbb{R} .
$$

Analogously we can prove

$$
\widehat{u}\left(x^{\prime}, z_{1},-\frac{1}{2}, z_{3}\right)-\widehat{u}\left(x^{\prime}, z_{1}, \frac{1}{2}, z_{3}\right)=0 \quad \text { a.e. }\left(x^{\prime}, z_{1}, z_{3}\right) \in \omega \times\left(\frac{-1}{2}, \frac{1}{2}\right) \times \mathbb{R} .
$$

These equalities prove the periodicity of $\widehat{u}$.

Step 3. Using the continuous embedding of $H^{1}\left(0,1 ; L^{2}(\omega)\right)$ into $L^{2}(\Gamma)$ and Lemma 5.3 (i), we deduce from (5.8) that $\bar{u}_{\varepsilon} / \varepsilon^{2}$ converges weakly to $\left(\tilde{u}^{\prime}\left(x^{\prime}, 0\right), 0\right)$ in $L^{2}(\omega)^{3}$. Thus, by (2.1) and (5.25), we get

$$
\frac{\widehat{u}_{\varepsilon}\left(x^{\prime}, z\right)}{\varepsilon^{2}} \rightarrow\left(\tilde{u}^{\prime}\left(x^{\prime}, 0\right), 0\right) \quad \text { in } L^{2}\left(\omega ; H^{1}\left(\widehat{Q}_{M}\right)\right)^{3} \quad \forall M>0 .
$$

Step 4 . Using the change of variables (5.17) in the equality $u_{\varepsilon} \nu=0$ on $\Gamma_{\varepsilon}$, we get

$$
-\frac{\delta_{\varepsilon}}{r_{\varepsilon}} \nabla \Psi\left(z^{\prime}\right) \widehat{u}_{\varepsilon}^{\prime}\left(x^{\prime}, z^{\prime},-\frac{\delta_{\varepsilon}}{r_{\varepsilon}} \Psi\left(z^{\prime}\right)\right)-\widehat{u}_{\varepsilon, 3}\left(x^{\prime}, z^{\prime},-\frac{\delta_{\varepsilon}}{r_{\varepsilon}} \Psi\left(z^{\prime}\right)\right)=0 \text {, a.e. in } \mathbb{R}^{2} \times Z^{\prime} .
$$

Thanks to (5.22) and (5.29), we then have

$$
\begin{aligned}
& \int_{\omega \times Z^{\prime}}\left|\frac{\delta_{\varepsilon}}{r_{\varepsilon}} \nabla \Psi\left(z^{\prime}\right) \widehat{u}_{\varepsilon}^{\prime}\left(x^{\prime}, z^{\prime}, 0\right)+\widehat{u}_{\varepsilon, 3}\left(x^{\prime}, z^{\prime}, 0\right)\right|^{2} d z^{\prime} d x^{\prime} \\
& \quad \leq C \frac{\delta_{\varepsilon}}{r_{\varepsilon}} \int_{\omega \times Z^{\prime}} \int_{\frac{-\delta_{\varepsilon}}{r_{\varepsilon}} \Psi\left(z^{\prime}\right)}^{0}\left|\frac{\delta_{\varepsilon}}{r_{\varepsilon}} \nabla \Psi\left(z^{\prime}\right) \partial_{z_{3}} \widehat{u}_{\varepsilon}^{\prime}\left(x^{\prime}, z^{\prime}, t\right)+\partial_{z_{3}} \widehat{u}_{\varepsilon, 3}\left(x^{\prime}, z^{\prime}, t\right)\right|^{2} d t d z^{\prime} d x^{\prime} \\
& \quad \leq C \frac{\delta_{\varepsilon}}{r_{\varepsilon}} \int_{\omega \times \widehat{Z}_{\varepsilon}}\left|\partial_{z_{3}} \widehat{u}_{\varepsilon}\right|^{2} d z d x^{\prime} \leq C \varepsilon^{3} \delta_{\varepsilon},
\end{aligned}
$$

Copyright $@$ by SIAM. Unauthorized reproduction of this article is prohibited. 
which implies

$$
\begin{aligned}
\int_{\omega \times Z^{\prime}} & \mid \frac{\delta_{\varepsilon}}{r_{\varepsilon}} \nabla \Psi\left(z^{\prime}\right) \widehat{u}_{\varepsilon}^{\prime}\left(x^{\prime}, z^{\prime}, 0\right)+\widehat{u}_{\varepsilon, 3}\left(x^{\prime}, z^{\prime}, 0\right) \\
& -\left.\int_{Z^{\prime}}\left(\frac{\delta_{\varepsilon}}{r_{\varepsilon}} \nabla \Psi\left(\tau^{\prime}\right) \widehat{u}_{\varepsilon}^{\prime}\left(x^{\prime}, \tau^{\prime}, 0\right)+\widehat{u}_{\varepsilon, 3}\left(x^{\prime}, \tau^{\prime}, 0\right)\right) d \tau^{\prime}\right|^{2} d x^{\prime} d z^{\prime} \leq C \varepsilon^{3} \delta_{\varepsilon} .
\end{aligned}
$$

Dividing by $\varepsilon^{3} r_{\varepsilon}$, using definition (5.25) of $\widehat{U}_{\varepsilon}$, and taking into account that $\nabla \Psi$ has null integral in $Z^{\prime}$ and (2.1), we get

$$
\begin{aligned}
& \int_{\omega \times Z^{\prime}}\left|\frac{\delta_{\varepsilon} \varepsilon^{\frac{1}{2}}}{r_{\varepsilon}^{\frac{3}{2}}} \nabla \Psi\left(z^{\prime}\right) \frac{\widehat{u}_{\varepsilon}^{\prime}\left(x^{\prime}, z^{\prime}, 0\right)}{\varepsilon^{2}}-\frac{\delta_{\varepsilon}}{r_{\varepsilon}} \int_{Z^{\prime}} \nabla \Psi\left(\tau^{\prime}\right) \widehat{U}_{\varepsilon}^{\prime}\left(x^{\prime}, \tau^{\prime}, 0\right) d \tau^{\prime}+\widehat{U}_{\varepsilon, 3}\left(x^{\prime}, z^{\prime}, 0\right)\right|^{2} d x^{\prime} d z^{\prime} \\
& \leq C \frac{\delta_{\varepsilon}}{r_{\varepsilon}} \rightarrow 0
\end{aligned}
$$

and then, by (5.26),

$$
\frac{\delta_{\varepsilon} \varepsilon^{\frac{1}{2}}}{r_{\varepsilon}^{\frac{3}{2}}} \nabla \Psi\left(z^{\prime}\right) \frac{\widehat{u}_{\varepsilon}^{\prime}\left(x^{\prime}, z^{\prime}, 0\right)}{\varepsilon^{2}} \rightarrow-\widehat{u}_{3}\left(x^{\prime}, z^{\prime}, 0\right) \text { in } L^{2}\left(\omega \times Z^{\prime}\right) .
$$

This convergence and (5.28) imply (5.18) and (5.19), depending on whether $\lambda$ is infinite or finite.

LeMmA 5.5. Let $p_{\varepsilon}^{1}$ be in $L^{2}\left(\Omega_{\varepsilon}\right)$ satisfying

$$
\left\|p_{\varepsilon}^{1}\right\|_{L^{2}\left(\Omega_{\varepsilon}\right)} \leq C \varepsilon^{\frac{3}{2}}
$$

and (assuming $p_{\varepsilon}$ extended by zero to $\Lambda_{\varepsilon}$ ) let us define $\widehat{p}_{\varepsilon}^{1}$ by

$$
\widehat{p}_{\varepsilon}^{1}\left(x^{\prime}, z\right)=p_{\varepsilon}^{1}\left(r_{\varepsilon} \kappa\left(\frac{x^{\prime}}{r_{\varepsilon}}\right)+r_{\varepsilon} z^{\prime}, r_{\varepsilon} z_{3}\right), \quad \text { a.e. }\left(x^{\prime}, z\right) \in \mathbb{R}^{2} \times \widehat{Z}_{\varepsilon} .
$$

Then there exists $\widehat{p}^{1} \in L^{2}(\omega \times \widehat{Q})$ such that, up to a subsequence,

$$
\frac{r_{\varepsilon}^{\frac{1}{2}}}{\varepsilon^{\frac{3}{2}}} \widehat{p}_{\varepsilon}^{1} \rightarrow \widehat{p}^{1} \text { in } L^{2}\left(\omega \times \widehat{Q}_{M}\right) \quad \forall M>0
$$

Proof. For every $M>0$, using the definition of $\widehat{p}_{\varepsilon}^{1}$ and (5.31), we deduce that for every $\varepsilon>0$ small enough (depending on $M$ ), we have

$$
\begin{gathered}
\int_{\omega \times \widehat{Q}_{M}}\left|\widehat{p}_{\varepsilon}^{1}\right|^{2} d x^{\prime} d z=\sum_{k^{\prime} \in \mathbb{Z}^{2}} r_{\varepsilon}^{2} \int_{\widehat{Q}_{M}}\left|p_{\varepsilon}^{1}\left(r_{\varepsilon}\left(k^{\prime}+z^{\prime}\right), r_{\varepsilon} z_{3}\right)\right|^{2} d z \\
\leq \frac{1}{r_{\varepsilon}} \sum_{k^{\prime} \in \mathbb{Z}^{2}} \int_{Q_{r_{\varepsilon}}^{k^{\prime}}}\left|p_{\varepsilon}^{1}(x)\right|^{2} d x=\frac{1}{r_{\varepsilon}} \int_{\Omega_{\varepsilon}}\left|p_{\varepsilon}^{1}\right|^{2} d x \leq C \frac{\varepsilon^{3}}{r_{\varepsilon}},
\end{gathered}
$$

and then there exists $\widehat{p}^{1}: \omega \times \widehat{Q} \longrightarrow \mathbb{R}$ such that (5.33) holds. By semicontinuity, (5.34) proves

$$
\int_{\omega \times \widehat{Q}_{M}}\left|\hat{p}^{1}\right|^{2} d x^{\prime} d z \leq C \quad \forall M>0,
$$

which shows that $\widehat{p}^{1}$ belongs to $L^{2}(\omega \times \widehat{Q})$.

Copyright $@$ by SIAM. Unauthorized reproduction of this article is prohibited. 
6. Obtaining the limit system and the corrector result. In this last section we use the results of the previous sections to prove Theorems 3.3, 3.4, and 3.8 describing the asymptotic behavior of the solution $\left(u_{\varepsilon}, p_{\varepsilon}\right)$ of the Navier-Stokes system (3.1).

Proof of Theorem 3.3. From (3.3) and $\operatorname{div} u_{\varepsilon}=0$ in $\Omega_{\varepsilon}$, Lemma 5.1 assures, up to a subsequence, the existence of $\tilde{u}^{\prime} \in H^{1}\left(0,1 ; L^{2}(\omega)\right)^{2}$ and $\tilde{w} \in H^{2}\left(0,1 ; H^{-1}(\omega)\right)$ satisfying (3.8) and the two last lines in (3.10). Moreover, using the decomposition $p_{\varepsilon}=p_{\varepsilon}^{0}+p_{\varepsilon}^{1}$ given by Theorem 3.1, defining $\tilde{p}_{\varepsilon}^{1} \in L^{2}\left(\tilde{\Omega}_{\varepsilon}\right)$ by

$$
\tilde{p}_{\varepsilon}^{1}(y)=p_{\varepsilon}^{1}\left(y^{\prime}, \varepsilon y_{3}\right), \text { a.e. } y \in \tilde{\Omega}_{\varepsilon},
$$

and taking into account (3.4), we deduce that, up to a subsequence, we have that there exist $\tilde{p} \in H^{1}(\omega)$, which has null mean value in $\omega$ (since $p_{\varepsilon}$ has null mean value in $\left.\Omega_{\varepsilon}\right)$, and $\tilde{p}_{1} \in L^{2}(\Omega)$, such that

$$
p_{\varepsilon}^{0} \rightarrow \tilde{p} \quad \text { in } H^{1}(\omega), \quad \frac{1}{\varepsilon} \tilde{p}_{\varepsilon}^{1} \rightarrow \tilde{p}^{1} \quad \text { in } L^{2}(\Omega),
$$

and as consequence

$$
\tilde{p}_{\varepsilon} \rightarrow \tilde{p} \quad \text { in } L^{2}(\Omega) \quad \frac{1}{\varepsilon} \partial_{y_{3}} \tilde{p}_{\varepsilon} \rightarrow \partial_{y_{3}} \tilde{p}^{1} \quad \text { in } L^{2}\left(\omega ; H^{-1}(0,1)\right),
$$

which in particular implies the first assertion in (3.9).

On the other hand, we remark that $\left(u_{\varepsilon}, p_{\varepsilon}\right)$ satisfies the variational equation

$$
\left\{\begin{array}{l}
\mu \int_{\Omega_{\varepsilon}} D u_{\varepsilon}: D \varphi_{\varepsilon} d x+\int_{\Omega_{\varepsilon}} \nabla_{x^{\prime}} p_{\varepsilon}^{0} \varphi_{\varepsilon}^{\prime} d x-\int_{\Omega_{\varepsilon}} p_{\varepsilon}^{1} \operatorname{div} \varphi_{\varepsilon} d x+\int_{\Omega_{\varepsilon}}\left(u_{\varepsilon} \cdot \nabla\right) u_{\varepsilon} \varphi_{\varepsilon} d x \\
+\frac{\gamma}{\varepsilon} \int_{\Gamma_{\varepsilon}} u_{\varepsilon} \varphi_{\varepsilon} d \sigma=\int_{\Omega_{\varepsilon}} f_{\varepsilon} \varphi_{\varepsilon} d x \\
\forall \varphi_{\varepsilon} \in H^{1}\left(\Omega_{\varepsilon}\right)^{3}, \varphi_{\varepsilon} \nu=0 \text { on } \Gamma_{\varepsilon}, \varphi_{\varepsilon}=0 \text { on } \partial \Omega_{\varepsilon} \backslash \Gamma_{\varepsilon}
\end{array}\right.
$$

The proof of Theorem 3.3 will be carried out using suitable test functions $\varphi_{\varepsilon}$ in (6.3).

Step 1. For $\tilde{\varphi}_{3} \in C_{c}^{1}(\Omega)$, we define $\varphi_{\varepsilon} \in H^{1}\left(\Omega_{\varepsilon}\right)^{3}$ by

$$
\varphi_{\varepsilon}^{\prime}(x)=0, \quad \varphi_{\varepsilon, 3}=\frac{1}{\varepsilon} \tilde{\varphi}_{3}\left(x^{\prime}, \frac{x_{3}}{\varepsilon}\right) \quad \forall x \in \Omega_{\varepsilon} .
$$

Then (6.3) gives

$$
\begin{aligned}
& \frac{\mu}{\varepsilon} \int_{\Omega_{\varepsilon}} \nabla_{x^{\prime}} u_{\varepsilon, 3}(x) \nabla_{y^{\prime}} \tilde{\varphi}_{3}\left(x^{\prime}, \frac{x_{3}}{\varepsilon}\right) d x+\frac{\mu}{\varepsilon^{2}} \int_{\Omega_{\varepsilon}} \partial_{x_{3}} u_{\varepsilon, 3}(x) \partial_{y_{3}} \tilde{\varphi}_{3}\left(x^{\prime}, \frac{x_{3}}{\varepsilon}\right) d x \\
& \quad-\frac{1}{\varepsilon^{2}} \int_{\Omega_{\varepsilon}} p_{\varepsilon}^{1}(x) \partial_{y_{3}} \tilde{\varphi}_{3}\left(x^{\prime}, \frac{x_{3}}{\varepsilon}\right) d x+\frac{1}{\varepsilon} \int_{\Omega_{\varepsilon}} u_{\varepsilon} \nabla u_{\varepsilon, 3}(x) \tilde{\varphi}_{\varepsilon, 3}\left(x^{\prime}, \frac{x_{3}}{\varepsilon}\right) d x \\
& =\frac{1}{\varepsilon} \int_{\Omega_{\varepsilon}} \tilde{f}_{\varepsilon, 3}\left(x^{\prime}, \frac{x_{3}}{\varepsilon}\right) \tilde{\varphi}_{3}\left(x^{\prime}, \frac{x_{3}}{\varepsilon}\right) d x
\end{aligned}
$$

Hölder's inequality, (3.3), (4.20), and $\left\|\tilde{\varphi}_{\varepsilon, 3}\right\|_{L^{\infty}(\Omega)} \leq C$ imply

$$
\left|\int_{\Omega_{\varepsilon}}\left(u_{\varepsilon} \nabla u_{\varepsilon, 3}\right) \tilde{\varphi}_{\varepsilon, 3} d x\right| \leq\left|\Omega_{\varepsilon}\right|^{\frac{1}{3}}\left\|u_{\varepsilon}\right\|_{L^{6}\left(\Omega_{\varepsilon}\right)}\left\|D u_{\varepsilon}\right\|_{L^{2}\left(\Omega_{\varepsilon}\right)^{3 \times 3}}\left\|\varphi_{\varepsilon, 3}\right\|_{L^{\infty}\left(\Omega_{\varepsilon}\right)} \leq C \varepsilon^{\frac{10}{3}} .
$$

Copyright (c) by SIAM. Unauthorized reproduction of this article is prohibited. 
Thus, using the change of variables $(3.5)$ and taking into account that $\operatorname{supp}\left(\tilde{\varphi}_{3}\right)$ is contained in $\Omega$, we get

$$
\begin{aligned}
& \mu \int_{\Omega} \nabla_{y^{\prime}} \tilde{u}_{\varepsilon, 3}(y) \nabla_{y^{\prime}} \tilde{\varphi}_{3}(y) d y+\frac{\mu}{\varepsilon^{2}} \int_{\Omega} \partial_{y_{3}} \tilde{u}_{\varepsilon, 3}(y) \partial_{y_{3}} \tilde{\varphi}_{3}(y) d y-\int_{\Omega} \frac{\tilde{p}_{\varepsilon}^{1}}{\varepsilon}(y) \partial_{y_{3}} \tilde{\varphi}_{3}(y) d y \\
& \quad=\int_{\Omega} \tilde{f}_{3}(y) \tilde{\varphi}_{3}(y) d y+O_{\varepsilon} .
\end{aligned}
$$

Passing to the limit in this inequality, thanks to (3.8) and (6.1), we deduce

$$
\partial_{y_{3}} \tilde{p}^{1}=\tilde{f}_{3} \quad \text { in } \Omega
$$

which combined with (6.2) proves the second assertion in (3.9).

Step 2. Case $\lambda \in(0,+\infty)$. This is the most difficult case and will be developed in more details. First, we remark that thanks to (3.3), div $u_{\varepsilon}=0$ in $\Omega_{\varepsilon}$, and (3.4), we can apply Lemmas 5.4 and 5.5 to deduce the existence of a function $\widehat{u} \in L^{2}\left(\omega ; \mathcal{V}^{3}\right)$, which satisfies (5.19) and (5.21), and a function $\widehat{p}^{1} \in L^{2}(\omega \times \widehat{Q})$ such that defining $\widehat{u}_{\varepsilon}$ and $\widehat{p}_{\varepsilon}^{1}$ by (5.16) and (5.32), respectively, convergences (5.20) and (5.33) hold, up to a subsequence.

For $\tilde{\varphi}^{\prime} \in C_{c}^{1}(\omega \times(-1,1))^{2}, \widehat{\varphi} \in C_{c}^{1}\left(\omega ; C_{\sharp}^{1}(\widehat{Q})\right)^{3}$ such that

$$
\left\{\begin{array}{l}
D_{z} \widehat{\varphi}\left(x^{\prime}, z\right)=0 \text { a.e. in }\left\{z_{3}>M\right\} \text { for some constant } M>0 \\
\tilde{\varphi}^{\prime}\left(y^{\prime}, y_{3}\right)=\tilde{\varphi}^{\prime}\left(y^{\prime}, 0\right) \text { if } y_{3} \leq 0, \quad \widehat{\varphi}\left(x^{\prime}, z^{\prime}, z_{3}\right)=\widehat{\varphi}\left(x^{\prime}, z^{\prime}, 0\right) \text { if } z_{3} \leq 0 \\
\lambda \nabla \Psi\left(z^{\prime}\right) \tilde{\varphi}^{\prime}\left(y^{\prime}, 0\right)+\widehat{\varphi}_{3}\left(y^{\prime}, z^{\prime}, 0\right)=0
\end{array}\right.
$$

and $\zeta \in C^{\infty}(\mathbb{R})$ satisfying

$$
\zeta(s)=1 \text { if } s<\frac{1}{3}, \quad \zeta(s)=0 \text { if } s>\frac{2}{3},
$$

we define $\varphi_{\varepsilon} \in H^{1}\left(\Omega_{\varepsilon}\right)^{3}$ by

$$
\left\{\begin{array}{l}
\varphi_{\varepsilon}^{\prime}(x)=\frac{1}{\varepsilon} \tilde{\varphi}^{\prime}\left(x^{\prime}, \frac{x_{3}}{\varepsilon}\right)+\frac{\delta_{\varepsilon}}{\lambda \varepsilon r_{\varepsilon}} \widehat{\varphi}^{\prime}\left(x^{\prime}, \frac{x}{r_{\varepsilon}}\right) \zeta\left(\frac{x_{3}}{\varepsilon}\right), \\
\varphi_{\varepsilon, 3}(x)=\frac{\delta_{\varepsilon}}{\lambda \varepsilon r_{\varepsilon}} \widehat{\varphi}_{3}\left(x^{\prime}, \frac{x}{r_{\varepsilon}}\right) \zeta\left(\frac{x_{3}}{\varepsilon}\right)-\frac{\delta_{\varepsilon}^{2}}{\lambda \varepsilon r_{\varepsilon}^{2}} \widehat{\varphi}^{\prime}\left(x^{\prime}, \frac{x}{r_{\varepsilon}}\right) \nabla \Psi\left(\frac{x^{\prime}}{r_{\varepsilon}}\right) \zeta\left(\frac{x_{3}}{r_{\varepsilon}}\right) .
\end{array}\right.
$$

Thanks to $\tilde{\varphi}^{\prime}(x)$ and $\widehat{\varphi}\left(x^{\prime}, z\right)$ equaling zero for $x^{\prime}$ outside a compact subset of $\omega$ and (6.5), the sequence $\varphi_{\varepsilon}$ satisfies that

$$
\varphi_{\varepsilon}=0 \text { on } \partial \Omega_{\varepsilon} \backslash \Gamma_{\varepsilon}, \quad \varphi_{\varepsilon} \nu=0 \text { on } \Gamma_{\varepsilon} .
$$

Thus, we can take such $\varphi_{\varepsilon}$ in (6.3). The problem is to pass to the limit in the different terms which appear in (6.3). Before, we remark that since $D_{z} \widehat{\varphi}=0$ a.e in $\left\{z_{3}>M\right\}$ and (6.6), we have

$$
\begin{gathered}
\varphi_{\varepsilon}(x)=\frac{1}{\varepsilon}\left(\tilde{\varphi}^{\prime}\left(x^{\prime}, \frac{x_{3}}{\varepsilon}\right), 0\right)+g_{\varepsilon} \text { in } \bar{\Omega}_{\varepsilon} \\
D \varphi_{\varepsilon}(x)=\frac{1}{\varepsilon^{2}} \sum_{i=1}^{2} \partial_{y_{3}} \tilde{\varphi}_{i}\left(x^{\prime}, \frac{x_{3}}{\varepsilon}\right) e_{i} \otimes e_{3}+\frac{\delta_{\varepsilon}}{\lambda \varepsilon r_{\varepsilon}^{2}} D_{z} \widehat{\varphi}\left(x^{\prime}, \frac{x}{r_{\varepsilon}}\right)+h_{\varepsilon}(x) \quad \text { in } \Omega_{\varepsilon}
\end{gathered}
$$

Copyright (c) by SIAM. Unauthorized reproduction of this article is prohibited. 
with $g_{\varepsilon} \in C^{0}\left(\bar{\Omega}_{\varepsilon}\right)^{3}, h_{\varepsilon} \in C^{0}\left(\bar{\Omega}_{\varepsilon}\right)^{3 \times 3}$ satisfying (thanks to $(2.1)$ and $\lambda \in(0, \infty)$ )

$$
\begin{gathered}
\varepsilon \int_{\Omega_{\varepsilon}}\left|g_{\varepsilon}\right|^{2} d x \leq C\left(\frac{\delta_{\varepsilon}^{2}}{r_{\varepsilon}^{2}}+\frac{\delta_{\varepsilon}^{4}}{\varepsilon r_{\varepsilon}^{3}}\right)=O_{\varepsilon}, \\
\varepsilon^{2} \int_{\Gamma_{\varepsilon}}\left|g_{\varepsilon}\right|^{2} d x \leq C \frac{\delta_{\varepsilon}^{2}}{r_{\varepsilon}^{2}}=O_{\varepsilon}, \\
\varepsilon^{3} \int_{\Omega_{\varepsilon}}\left|h_{\varepsilon}\right|^{2} d x \leq C \varepsilon^{3}\left(\frac{\delta_{\varepsilon}^{2}}{\varepsilon r_{\varepsilon}^{2}}+\frac{\delta_{\varepsilon}^{2}}{\varepsilon^{3} r_{\varepsilon}^{2}}+\frac{\delta_{\varepsilon}^{4}}{\varepsilon^{2} r_{\varepsilon}^{5}}+\frac{1}{\varepsilon}\right)=O_{\varepsilon} .
\end{gathered}
$$

- First term in (6.3). Thanks to (3.3), (6.8), and (6.11), we easily have

$$
\begin{aligned}
\mu \int_{\Omega_{\varepsilon}} D u_{\varepsilon}(x): D \varphi_{\varepsilon}(x) d x & =\frac{\mu}{\varepsilon^{2}} \int_{\Omega_{\varepsilon}^{+}} \partial_{x_{3}} u_{\varepsilon}^{\prime}(x) \partial_{y_{3}} \tilde{\varphi}^{\prime}\left(x^{\prime}, \frac{x_{3}}{\varepsilon}\right) d x \\
& +\mu \frac{\delta_{\varepsilon}}{\lambda \varepsilon r_{\varepsilon}^{2}} \int_{\Omega_{\varepsilon}^{+}} D u_{\varepsilon}(x): D_{z} \widehat{\varphi}\left(x^{\prime}, \frac{x}{r_{\varepsilon}}\right) d x+O_{\varepsilon} .
\end{aligned}
$$

For the first term on the right-hand side of this equality, we use the change of variables (3.5) and (3.8), which gives

$$
\frac{1}{\varepsilon^{2}} \int_{\Omega_{\varepsilon}^{+}} \partial_{x_{3}} u_{\varepsilon}^{\prime}(x) \partial_{y_{3}} \tilde{\varphi}^{\prime}\left(x^{\prime}, \frac{x_{3}}{\varepsilon}\right) d x=\frac{1}{\varepsilon^{2}} \int_{\Omega} \partial_{y_{3}} \tilde{u}_{\varepsilon}^{\prime} \partial_{y_{3}} \tilde{\varphi}^{\prime} d y=\int_{\Omega} \partial_{y_{3}} \tilde{u}^{\prime} \partial_{y_{3}} \tilde{\varphi}^{\prime} d y+O_{\varepsilon} .
$$

Analogously, using the change of variables (5.17), the assumptions on the support of $D_{z} \widehat{\varphi}$ and (5.20), we get

$$
\begin{aligned}
\frac{\delta_{\varepsilon}}{\lambda \varepsilon r_{\varepsilon}^{2}} \int_{\Omega_{\varepsilon}^{+}} D u_{\varepsilon}(x): D_{z} \widehat{\varphi}\left(x^{\prime}, \frac{x}{r_{\varepsilon}}\right) d x & =\frac{\delta_{\varepsilon} \varepsilon^{\frac{1}{2}}}{\lambda r_{\varepsilon}^{\frac{3}{2}}} \int_{\omega \times \widehat{Q}_{M}} D_{z}\left(\frac{\widehat{u}_{\varepsilon}}{\varepsilon^{\frac{1}{2}} r_{\varepsilon}^{\frac{1}{2}}}\right): D_{z} \widehat{\varphi} d x^{\prime} d z \\
& =\int_{\omega \times \widehat{Q}} D_{z} \widehat{u}: D_{z} \widehat{\varphi} d x^{\prime} d z+O_{\varepsilon} .
\end{aligned}
$$

Therefore, (6.12) can be written as (6.13)

$$
\mu \int_{\Omega_{\varepsilon}} D u_{\varepsilon}(x): D \varphi_{\varepsilon}(x) d x=\mu \int_{\Omega} \partial_{y_{3}} \tilde{u}^{\prime} \partial_{y_{3}} \tilde{\varphi}^{\prime} d y+\mu \int_{\omega \times \widehat{Q}} D_{z} \widehat{u}: D_{z} \widehat{\varphi} d x^{\prime} d z+O_{\varepsilon} .
$$

- Second term in (6.3). Thanks to (3.4), (6.7), (6.9), (3.5), and (6.1) we get (6.14)

$\int_{\Omega_{\varepsilon}} \nabla_{x^{\prime}} p_{\varepsilon}^{0}\left(x^{\prime}\right) \varphi_{\varepsilon}^{\prime}(x) d x=\frac{1}{\varepsilon} \int_{\Omega_{\varepsilon}^{+}} \nabla_{x^{\prime}} \tilde{p}_{\varepsilon}^{0}\left(x^{\prime}\right) \tilde{\varphi}^{\prime}\left(x^{\prime}, \frac{x_{3}}{\varepsilon}\right) d x+O_{\varepsilon}=\int_{\Omega} \nabla_{y^{\prime}} \tilde{p}\left(y^{\prime}\right) \tilde{\varphi}^{\prime}(y) d y+O_{\varepsilon}$.

- Third term in (6.3). Using (3.4), (6.8), (6.11), the change of variables (5.17), and (5.33), we obtain

$$
\begin{aligned}
& \int_{\Omega_{\varepsilon}} p_{\varepsilon}^{1}(x) \operatorname{div} \varphi_{\varepsilon}(x) d x=\frac{\delta_{\varepsilon}}{\lambda \varepsilon r_{\varepsilon}^{2}} \int_{\Omega_{\varepsilon}^{+}} p_{\varepsilon}^{1}(x) \operatorname{div}_{z} \widehat{\varphi}\left(x^{\prime}, \frac{x}{r_{\varepsilon}}\right) d x+O_{\varepsilon} \\
& =\frac{\delta_{\varepsilon} \varepsilon^{\frac{1}{2}}}{\lambda r_{\varepsilon}^{\frac{3}{2}}} \int_{\omega \times \widehat{Q}_{M}}\left(\frac{r_{\varepsilon}^{\frac{1}{2}}}{\varepsilon^{\frac{3}{2}}} p_{\varepsilon}^{1}\right) \operatorname{div}_{z} \widehat{\varphi} d x^{\prime} d z+O_{\varepsilon}=\int_{\omega \times \widehat{Q}} \widehat{p}^{1} \operatorname{div}_{z} \widehat{\varphi} d x^{\prime} d z+O_{\varepsilon} .
\end{aligned}
$$

Copyright $@$ by SIAM. Unauthorized reproduction of this article is prohibited. 
- Fourth term in (6.3). Using (3.3), (4.20), and $\left\|\varphi_{\varepsilon}\right\|_{L^{\infty}\left(\Omega_{\varepsilon}\right)^{3}} \leq C / \varepsilon$, we get

$$
\int_{\Omega_{\varepsilon}}\left(u_{\varepsilon} \cdot \nabla\right) u_{\varepsilon} \varphi_{\varepsilon} d x=O_{\varepsilon} .
$$

- Fifth term in (6.3). Thanks to $u_{\varepsilon}\left(x^{\prime}, \varepsilon\right)=0$ in $\omega$ and (3.3), we have that

$$
\int_{\Gamma_{\varepsilon}}\left|u_{\varepsilon}\right|^{2} d \sigma \leq C \varepsilon \int_{\Omega_{\varepsilon}}\left|D u_{\varepsilon}\right|^{2} d x \leq C \varepsilon^{4} .
$$

So, taking into account $(6.7),(6.10)$, and $\tilde{\varphi}^{\prime}(y)=\tilde{\varphi}^{\prime}\left(y^{\prime}, 0\right)$ a.e. in $y_{3} \leq 0$, we have

$$
\begin{aligned}
\frac{\gamma}{\varepsilon} \int_{\Gamma_{\varepsilon}} u_{\varepsilon} \varphi_{\varepsilon} d \sigma & =\frac{\gamma}{\varepsilon^{2}} \int_{\omega} u_{\varepsilon}^{\prime}\left(x^{\prime},-\delta_{\varepsilon} \Psi\left(\frac{x^{\prime}}{r_{\varepsilon}}\right)\right) \tilde{\varphi}^{\prime}\left(x^{\prime}, 0\right) \sqrt{1+\frac{\delta_{\varepsilon}^{2}}{r_{\varepsilon}^{2}}\left|\nabla \Psi\left(\frac{x^{\prime}}{r_{\varepsilon}}\right)\right|^{2}} d x^{\prime}+O_{\varepsilon} \\
& =\frac{\gamma}{\varepsilon^{2}} \int_{\omega} u_{\varepsilon}^{\prime}\left(x^{\prime},-\delta_{\varepsilon} \Psi\left(\frac{x^{\prime}}{r_{\varepsilon}}\right)\right) \tilde{\varphi}^{\prime}\left(x^{\prime}, 0\right) d x^{\prime}+O_{\varepsilon} .
\end{aligned}
$$

However, integrating in the $x_{3}$ variable, we have

$$
\int_{\omega}\left|u_{\varepsilon}\left(x^{\prime},-\delta_{\varepsilon} \Psi\left(\frac{x^{\prime}}{r_{\varepsilon}}\right)\right)-u_{\varepsilon}\left(x^{\prime}, 0\right)\right|^{2} d x^{\prime} \leq C \delta_{\varepsilon} \int_{\Omega_{\varepsilon}}\left|D u_{\varepsilon}\right|^{2} d x \leq C \delta_{\varepsilon} \varepsilon^{3},
$$

and so, using that $u_{\varepsilon}\left(x^{\prime}, 0\right)=\tilde{u}_{\varepsilon}\left(x^{\prime}, 0\right)$ in $\omega$ and $(3.8)$, we get

$$
\frac{\gamma}{\varepsilon} \int_{\Gamma_{\varepsilon}} u_{\varepsilon} \varphi_{\varepsilon} d \sigma=\frac{\gamma}{\varepsilon^{2}} \int_{\omega} \tilde{u}_{\varepsilon}^{\prime}\left(y^{\prime}, 0\right) \tilde{\varphi}^{\prime}\left(y^{\prime}, 0\right) d y^{\prime}+O_{\varepsilon}=\gamma \int_{\omega} \tilde{u}^{\prime}\left(y^{\prime}, 0\right) \tilde{\varphi}^{\prime}\left(y^{\prime}, 0\right) d y^{\prime}+O_{\varepsilon} .
$$

- Sixth term in (6.3). Thanks to (6.7), (6.9), and the change of variables (3.5), we get

$$
\int_{\Omega_{\varepsilon}} f_{\varepsilon}(x) \varphi_{\varepsilon}(x) d x=\frac{1}{\varepsilon} \int_{\Omega_{\varepsilon}^{+}} \tilde{f}^{\prime}\left(x^{\prime}, \frac{x_{3}}{\varepsilon}\right) \varphi_{\varepsilon}^{\prime}\left(x^{\prime}, \frac{x_{3}}{\varepsilon}\right) d x+O_{\varepsilon}=\int_{\Omega} \tilde{f}^{\prime} \tilde{\varphi}^{\prime} d y+O_{\varepsilon} .
$$

From (6.13)-(6.19) we then deduce that $\tilde{u}^{\prime}, \tilde{p}, \widehat{u}$, and $\widehat{p}^{1}$ satisfy

$$
\begin{aligned}
& \mu \int_{\Omega} \partial_{y_{3}} \tilde{u}^{\prime}(y) \partial_{y_{3}} \tilde{\varphi}^{\prime}(y) d y+\mu \int_{\omega} \int_{\widehat{Q}} D_{z} \widehat{u}\left(x^{\prime}, z\right): D_{z} \widehat{\varphi}\left(x^{\prime}, z\right) d z d x^{\prime}+\int_{\Omega} \nabla_{y^{\prime}} \tilde{p}\left(y^{\prime}\right) \tilde{\varphi}^{\prime}(y) d y \\
& -\int_{\omega} \int_{\widehat{Q}} \hat{p}^{1}\left(x^{\prime}, z\right) \operatorname{div}_{z} \widehat{\varphi}\left(x^{\prime}, z\right) d z d x^{\prime}+\gamma \int_{\Gamma} \tilde{u}^{\prime} \tilde{\varphi}^{\prime} d \sigma=\int_{\Omega} \tilde{f}^{\prime}(y) \tilde{\varphi}^{\prime}(y) d y
\end{aligned}
$$

for every $\tilde{\varphi}^{\prime} \in C_{c}^{1}(\omega \times(-1,1))^{2}, \widehat{\varphi} \in C_{c}^{1}\left(\omega ; C_{\sharp}^{1}(\widehat{Q})\right)^{3}$ such that (6.5) is satisfied. By density, this equality holds true for every $\tilde{\varphi}^{\prime} \in H^{1}\left(0,1 ; L^{2}(\omega)\right)^{2}$, and every $\widehat{\varphi} \in$ $L^{2}(\omega ; \mathcal{V})^{3}$ such that

$$
\tilde{\varphi}^{\prime}\left(x^{\prime}, 1\right)=0 \text {, a.e. } x^{\prime} \in \omega, \quad \lambda \nabla \Psi\left(z^{\prime}\right) \tilde{\varphi}^{\prime}\left(x^{\prime}, 0\right)+\widehat{\varphi}_{3}\left(x^{\prime}, z^{\prime}, 0\right)=0 \text {, a.e. }\left(x^{\prime}, z^{\prime}\right) \in \omega \times Z^{\prime} .
$$

Let us now obtain a problem for $\tilde{u}^{\prime}$ and $\tilde{p}$ eliminating $\widehat{u}$ and $\widehat{p}^{1}$ in (6.20). For this purpose, taking $\tilde{\varphi}^{\prime}=0$ in $(6.20)$, we deduce that $\left(\widehat{u}, \widehat{p}^{1}\right)$ is a solution of

$$
\left\{\begin{array}{l}
-\mu \Delta_{z} \widehat{u}+\nabla_{z} \widehat{p}^{1}=0 \text { in } \mathbb{R}^{2} \times \mathbb{R}^{+}, \\
\operatorname{div}_{z} \widehat{u}=0 \text { in } \mathbb{R}^{2} \times \mathbb{R}^{+}, \\
\left(\widehat{u}, \widehat{p}^{1}\right) \in \mathcal{V}^{3} \times L_{\sharp}^{2}(\widehat{Q}), \\
\widehat{u}_{3}\left(x^{\prime}, z^{\prime}, 0\right)=-\lambda \nabla \Psi\left(z^{\prime}\right) \tilde{u}^{\prime}\left(x^{\prime}, 0\right) \text { on } \mathbb{R}^{2} \times\{0\}, \\
\partial_{z_{3}} \widehat{u}^{\prime}=0 \text { on } \mathbb{R}^{2} \times\{0\},
\end{array}\right.
$$

Copyright (c) by SIAM. Unauthorized reproduction of this article is prohibited. 
a.e. $x^{\prime}$ in $\omega$. Defining $\left(\widehat{\phi}^{i}, \widehat{q}^{i}\right), i=1,2$, by (3.13), we deduce by linearity and uniqueness that

(6.22)

$$
\begin{aligned}
& D_{z} \widehat{u}\left(x^{\prime}, z\right)=-\lambda\left(\tilde{u}_{1}\left(x^{\prime}, 0\right) D_{z} \widehat{\phi}^{1}(z)+\tilde{u}_{2}\left(x^{\prime}, 0\right) D_{z} \widehat{\phi}^{2}(z)\right), \text { a.e. in } \mathbb{R}^{2} \times \mathbb{R}^{+}, \\
& \widehat{p}^{1}\left(x^{\prime}, z\right)=\lambda\left(\tilde{u}_{1}\left(x^{\prime}, 0\right) \widehat{q}^{1}(z)+\tilde{u}_{2}\left(x^{\prime}, 0\right) \widehat{q}^{2}(z)\right), \text { a.e. in } \mathbb{R}^{2} \times \mathbb{R}^{+} .
\end{aligned}
$$

Now, taking in (6.20), $\tilde{\varphi}^{\prime} \in H^{1}\left(0,1 ; L^{2}(\omega)\right)^{2}, \tilde{\varphi}^{\prime}\left(x^{\prime}, 1\right)=0$, a.e. $x^{\prime} \in \omega$, choosing $\widehat{\varphi}$ as

$$
\widehat{\varphi}\left(x^{\prime}, z\right)=-\lambda\left(\tilde{\varphi}_{1}\left(x^{\prime}, 0\right) \widehat{\phi}^{1}(z)+\tilde{\varphi}_{2}\left(x^{\prime}, 0\right) \widehat{\phi}^{2}(z)\right)
$$

and using (6.22), we get

$$
\begin{aligned}
& \mu \int_{\Omega} \partial_{y_{3}} \tilde{u}^{\prime} \partial_{y_{3}} \tilde{\varphi}^{\prime} d y+\int_{\Omega} \nabla_{y^{\prime}} \tilde{p} \tilde{\varphi}^{\prime} d y+\lambda^{2} \int_{\omega} R \tilde{u}^{\prime}\left(y^{\prime}, 0\right) \tilde{\varphi}^{\prime}\left(y^{\prime}, 0\right) d y^{\prime} \\
& \quad+\gamma \int_{\Gamma} \tilde{u}^{\prime} \tilde{\varphi}^{\prime} d \sigma=\int_{\Omega} \tilde{f}^{\prime} \tilde{\varphi}^{\prime} d y
\end{aligned}
$$

with $R \in \mathbb{R}^{2 \times 2}$ defined by (3.14). By the arbitrariness of $\tilde{\varphi}^{\prime}$ we then deduce that $\tilde{u}^{\prime}$, $\tilde{w}$, and $\tilde{p}$ are a solution of (3.10) and (3.15).

Step 3. Case $\lambda=0$. As in the previous step, we consider $\tilde{\varphi}^{\prime} \in C_{c}^{1}(\omega \times(-1,1))^{2}$, with $\tilde{\varphi}^{\prime}(y)=\tilde{\varphi}^{\prime}\left(y^{\prime}, 0\right)$ if $y_{3} \leq 0$. Then, for $\zeta \in C^{\infty}(\mathbb{R})$ satisfying (6.6), we define $\varphi_{\varepsilon} \in H^{1}\left(\Omega_{\varepsilon}\right)^{3}$ by

$$
\varphi_{\varepsilon}^{\prime}(x)=\frac{1}{\varepsilon} \tilde{\varphi}^{\prime}\left(x^{\prime}, \frac{x_{3}}{\varepsilon}\right), \quad \varphi_{\varepsilon, 3}(x)=-\frac{\delta_{\varepsilon}}{\varepsilon r_{\varepsilon}} \zeta\left(\frac{x_{3}}{r_{\varepsilon}}\right) \tilde{\varphi}^{\prime}\left(x^{\prime}, 0\right) \nabla \Psi\left(\frac{x^{\prime}}{r_{\varepsilon}}\right) .
$$

For every $\varepsilon>0$, the function $\varphi_{\varepsilon}$ satisfies $\varphi_{\varepsilon}=0$ on $\partial \Omega_{\varepsilon} \backslash \Gamma_{\varepsilon}, \varphi_{\varepsilon} \nu=0$ on $\Gamma_{\varepsilon}$. So, we can choose such $\varphi_{\varepsilon}$ in (6.3). Taking into account that, thanks to $\lambda=0$, we have

$$
\begin{aligned}
& \lim _{\varepsilon \rightarrow 0}\left(\varepsilon^{3} \int_{\Omega_{\varepsilon}}\left|D \varphi_{\varepsilon}(x)-\sum_{i=1}^{2} \partial_{y_{3}} \tilde{\varphi}_{i}\left(x^{\prime}, \frac{x_{3}}{\varepsilon}\right) e_{i} \otimes e_{3}\right|^{2} d x\right)=0, \\
& \lim _{\varepsilon \rightarrow 0}\left(\varepsilon \int_{\Omega_{\varepsilon}}\left|\varphi_{\varepsilon, 3}(x)\right|^{2} d x\right)=0,
\end{aligned}
$$

and (3.8), (6.1), it is simple to pass to the limit in (6.3) to deduce that $\tilde{u}^{\prime}, \tilde{p}$ satisfy

$$
\mu \int_{\Omega} \partial_{y_{3}} \tilde{u}^{\prime} \partial_{y_{3}} \tilde{\varphi}^{\prime} d y+\int_{\Omega} \nabla_{y^{\prime}} \tilde{p} \tilde{\varphi}^{\prime} d y+\gamma \int_{\Gamma} \tilde{u}^{\prime} \tilde{\varphi}^{\prime} d \sigma=\int_{\Omega} \tilde{f}^{\prime} \tilde{\varphi}^{\prime} d y
$$

for every $\tilde{\varphi}^{\prime}$ as above, which implies that $\tilde{u}^{\prime}, \tilde{w}, \tilde{p}$ satisfy (3.10) and (3.16).

Step 4. Case $\lambda=+\infty$. We now consider $\tilde{\varphi}^{\prime} \in C_{c}^{\infty}(\omega \times(-1,1))^{2}$, with $\tilde{\varphi}^{\prime}(y)=$ $\tilde{\varphi}^{\prime}\left(y^{\prime}, 0\right)$ if $y_{3} \leq 0$ and satisfying the boundary condition $\tilde{\varphi}^{\prime} \in W^{\perp}$ on $\Gamma$, i.e.,

$$
\tilde{\varphi}^{\prime}\left(y^{\prime}, 0\right) \nabla \Psi\left(z^{\prime}\right)=0 \text {, a.e. }\left(y^{\prime}, z^{\prime}\right) \in \omega \times Z^{\prime} .
$$

Observe that this choice of $\tilde{\varphi}^{\prime}$ implies that $\varphi_{\varepsilon}$ defined by

$$
\varphi_{\varepsilon}^{\prime}(x)=\frac{1}{\varepsilon} \tilde{\varphi}^{\prime}\left(x^{\prime}, \frac{x_{3}}{\varepsilon}\right), \quad \varphi_{\varepsilon, 3}(x)=0,
$$

Copyright $@$ by SIAM. Unauthorized reproduction of this article is prohibited. 
satisfies $\varphi_{\varepsilon}=0$ on $\partial \Omega_{\varepsilon} \backslash \Gamma_{\varepsilon}, \varphi_{\varepsilon} \nu=0$ on $\Gamma_{\varepsilon}$. Taking such $\varphi_{\varepsilon}$ in (6.3) and reasoning as above, we can pass to the limit to deduce that (6.24) holds for such $\tilde{\varphi}^{\prime}$. When the dimension of the space $W$ defined by (3.11) is zero or two, it is clear, reasoning by density, that this implies that $\tilde{u}^{\prime}, \tilde{w}, \tilde{p}$ are a solution of (3.10) and (3.12). When the dimension of $W$ is one, we can reason as follows. We consider a unitary vector $\xi^{\prime} \in \mathbb{R}^{2}$ generating $W$. Then, for every $\tilde{\varphi}^{\prime} \in C_{c}^{\infty}(\omega \times(-1,1))^{2}$, with $\tilde{\varphi}^{\prime}(y)=\tilde{\varphi}^{\prime}\left(y^{\prime}, 0\right)$ if $y_{3} \leq 0$, the function $\tilde{\phi}^{\prime} \in C_{c}^{\infty}(\omega \times(-1,1))^{2}$ defined by

$$
\tilde{\phi}^{\prime}(y)=\tilde{\varphi}^{\prime}(y)-\left(\tilde{\varphi}^{\prime}\left(y^{\prime}, 0\right) \xi\right) \xi \quad \forall y \in \omega \times(-1,1)
$$

satisfies $\tilde{\phi}^{\prime}(y)=\tilde{\phi}^{\prime}\left(y^{\prime}, 0\right)$ if $y_{3} \leq 0$ and the boundary condition $\tilde{\phi}^{\prime} \in W^{\perp}$ on $\Gamma$. By the above proved results, this shows that (6.24) holds for $\tilde{\varphi}^{\prime}$ replaced by $\tilde{\phi}^{\prime}$, which taking into account the definition of $\tilde{\phi}^{\prime}$ gives

$$
\begin{aligned}
& \mu \int_{\Omega} \partial_{y_{3}} \tilde{u}^{\prime} \partial_{y_{3}} \tilde{\varphi}^{\prime} d y+\int_{\Omega} \nabla_{y^{\prime}} \tilde{p}\left(\tilde{\varphi}^{\prime}-\left(\tilde{\varphi}^{\prime}\left(y^{\prime}, 0\right) \xi\right) \xi\right) d y \\
& \quad+\gamma \int_{\Gamma} \tilde{u}^{\prime}\left(\tilde{\varphi}^{\prime}-\left(\tilde{\varphi}^{\prime} \xi\right) \xi\right) d \sigma=\int_{\Omega} \tilde{f}^{\prime}\left(\tilde{\varphi}^{\prime}-\left(\tilde{\varphi}^{\prime}\left(y^{\prime}, 0\right) \xi\right) \xi\right) d y .
\end{aligned}
$$

By density, this equality holds true for every $\tilde{\varphi}^{\prime} \in H^{1}(\Omega)^{2}$, with $\tilde{\varphi}^{\prime}=0$ on $\partial \Omega \backslash \Gamma$. In the particular case where $\tilde{\varphi}^{\prime} \in W^{\perp}$ on $\Gamma$, we have that $\tilde{\varphi}\left(y^{\prime}, 0\right) \xi=0$ in $\omega$, and then (6.26) proves that (6.24) holds for such $\tilde{\varphi}^{\prime}$; i.e., $\tilde{u}^{\prime}, \tilde{w}, \tilde{p}$ are a solution of (3.10) and (3.12).

Proof of Theorem 3.4. To simplify the exposition let us consider only the case $\lambda \in(0,+\infty)$. The other cases are obtained by proceeding similarly.

Integrating once with respect to $y_{3}$ the homogenized system (3.10), taking into account that both $\tilde{p}$ and $\tilde{f}^{\prime}$ do not depend on the variable $y_{3}$, and using the boundary condition (3.15) on $\Gamma$, we get

$$
-\mu \partial_{y_{3}} \tilde{u}^{\prime}(y)=\left(\tilde{f}^{\prime}\left(y^{\prime}\right)-\nabla_{y^{\prime}} \tilde{p}\left(y^{\prime}\right)\right) y_{3}-\left(\gamma I+\lambda^{2} R\right) \tilde{u}^{\prime}\left(y^{\prime}, 0\right), \quad \text { a.e. } y \in \Omega .
$$

Integrating again (6.27) with respect to $y_{3}$, we have

$$
-\mu \tilde{u}^{\prime}(y)=\frac{1}{2}\left(\tilde{f}^{\prime}\left(y^{\prime}\right)-\nabla_{y^{\prime}} \tilde{p}\left(y^{\prime}\right)\right) y_{3}^{2}-\left(\gamma I+\lambda^{2} R\right) \tilde{u}^{\prime}\left(y^{\prime}, 0\right) y_{3}+C,
$$

a.e. $y \in \Omega$, which for $y_{3}=0$ gives that $\tilde{u}\left(y^{\prime}, 0\right)=-\frac{C}{\mu}$, and so we have

$$
\tilde{u}^{\prime}(y)=\frac{-1}{2 \mu}\left(\tilde{f}^{\prime}\left(y^{\prime}\right)-\nabla_{y^{\prime}} \tilde{p}\left(y^{\prime}\right)\right) y_{3}^{2}-\frac{1}{\mu}\left(I+\left(\frac{\gamma}{\mu} I+\frac{\lambda^{2}}{\mu} R\right) y_{3}\right) C .
$$

Now, using that $\tilde{u}^{\prime}\left(y^{\prime}, 1\right)=0$, a.e. in $\omega$, we get

$$
C=-\frac{1}{2}\left(\left(1+\frac{\gamma}{\mu}\right) I+\frac{\lambda^{2}}{\mu} R\right)^{-1}\left(\tilde{f}^{\prime}\left(y^{\prime}\right)-\nabla_{y^{\prime}} \tilde{p}\left(y^{\prime}\right)\right) .
$$

Substituting this expression into (6.28), we then get

$$
\begin{aligned}
\tilde{u}^{\prime}(y)= & \frac{-1}{2 \mu}\left(\tilde{f}^{\prime}\left(y^{\prime}\right)-\nabla_{y^{\prime}} \tilde{p}\left(y^{\prime}\right)\right) y_{3}^{2} \\
& +\frac{1}{2 \mu}\left(I+\left(\frac{\gamma}{\mu} I+\frac{\lambda^{2}}{\mu} R\right) y_{3}\right)\left(\left(1+\frac{\gamma}{\mu}\right) I+\frac{\lambda^{2}}{\mu} R\right)^{-1}\left(\tilde{f}^{\prime}\left(y^{\prime}\right)-\nabla_{y^{\prime}} \tilde{p}\left(y^{\prime}\right)\right) \\
= & \frac{\left(y_{3}-1\right)}{2 \mu}\left(y_{3} I+\left(\left(1+\frac{\gamma}{\mu}\right) I+\frac{\lambda^{2}}{\mu} R\right)^{-1}\right)\left(\nabla_{y^{\prime}} \tilde{p}\left(y^{\prime}\right)-\tilde{f}^{\prime}\left(y^{\prime}\right)\right),
\end{aligned}
$$

Copyright (c) by SIAM. Unauthorized reproduction of this article is prohibited. 
a.e. $y \in \Omega$, which agrees with (3.21).

Substituting (3.21) into the second equation in (3.10) and integrating in $(0,1)$ with respect to $y_{3}$, we deduce that $\tilde{p}$ satisfies the Reynolds equation which appears in (3.20). The boundary condition for $\tilde{p}$ in (3.20) just follows using in (see (3.10))

$$
\int_{0}^{1} \tilde{u}^{\prime}(y) d y_{3} \nu=0 \quad \text { on } \partial \omega
$$

the expression of $\tilde{u}^{\prime}$ given by (3.21). Finally, expression (3.19) of $\tilde{w}$ is a simple consequence of the second equation in $(3.10)$ and $\tilde{w}\left(y^{\prime}, 0\right)=0$ in $\omega$.

It remains to prove Theorem 3.8; we will use the following lemma.

Lemma 6.1. For $\varphi_{\varepsilon} \in H^{1}\left(\Omega_{\varepsilon}\right)^{3}, \varphi_{\varepsilon} \nu=0$ on $\Gamma_{\varepsilon}, \varphi_{\varepsilon}=0$ on $\partial \Omega_{\varepsilon} \backslash \Gamma_{\varepsilon}$, and such that there exists $C>0$ satisfying

$$
f_{\Omega_{\varepsilon}}\left|D \varphi_{\varepsilon}\right|^{2} d x \leq C \varepsilon^{2}, \quad f_{\Omega_{\varepsilon}}\left|\operatorname{div} \varphi_{\varepsilon}\right|^{2} d x \leq C \varepsilon^{4} \quad \forall \varepsilon>0,
$$

we define $\tilde{\varphi}_{\varepsilon} \in H^{1}\left(\tilde{\Omega}_{\varepsilon}\right)^{3}$ by $\tilde{\varphi}_{\varepsilon}(y)=\varphi_{\varepsilon}\left(y^{\prime}, \varepsilon y_{3}\right)$ a.e. $y \in \Omega$. Assuming that there exist $\tilde{\varphi}^{\prime} \in H^{1}\left(0,1 ; L^{2}(\omega)\right)^{2}, \tilde{\rho} \in H^{1}\left(0,1 ; H^{-1}(\omega)\right)$, and $\tilde{\xi} \in L^{2}(\Omega)$ satisfying

$$
\begin{gathered}
\tilde{\varphi}^{\prime}(1)=0 \text { in } L^{2}(\omega), \quad \tilde{\rho}(0)=\tilde{\rho}(1)=0 \text { in } H^{-1}(\omega), \\
\operatorname{div}_{y^{\prime}} \tilde{\varphi}^{\prime}+\partial_{y_{3}} \tilde{\rho}=\tilde{\xi} \text { in } L^{2}\left(0,1 ; H^{-1}(\omega)\right),
\end{gathered}
$$

such that

$$
\left\{\begin{array}{l}
\frac{\tilde{\varphi}_{\varepsilon}}{\varepsilon} \rightarrow 0 \text { in } H^{1}(\Omega)^{3}, \frac{\tilde{\varphi}_{\varepsilon}}{\varepsilon^{2}} \rightarrow\left(\tilde{\varphi}^{\prime}, 0\right) \text { in } H^{1}\left(0,1 ; L^{2}(\omega)\right)^{3}, \\
\frac{\tilde{\varphi}_{\varepsilon, 3}}{\varepsilon^{3}} \rightarrow \tilde{\rho} \text { in } H^{1}\left(0,1 ; H^{-1}(\omega)\right), \\
\frac{1}{\varepsilon^{2}} \operatorname{div}_{y^{\prime}} \tilde{\varphi}_{\varepsilon}^{\prime}+\frac{1}{\varepsilon^{3}} \partial_{y_{3}} \tilde{\varphi}_{\varepsilon, 3} \rightarrow \tilde{\xi} \text { in } L^{2}\left(0,1 ; H^{-1}(\omega)\right),
\end{array}\right.
$$

we then have that, defining $\tilde{u}^{\prime} \in H^{1}\left(0,1 ; L^{2}(\omega)\right)^{2}, \tilde{p} \in H^{1}(\Omega)$ by Theorem 3.3, the solution $\left(u_{\varepsilon}, p_{\varepsilon}\right)$ of $(3.1)$ satisfies the following:

(i) If $\lambda=0,+\infty$,

$$
\begin{aligned}
& \lim _{\varepsilon \rightarrow 0}\left(\frac{\mu}{\varepsilon^{3}} \int_{\Omega_{\varepsilon}} D u_{\varepsilon}: D \varphi_{\varepsilon} d x-\frac{1}{\varepsilon^{3}} \int_{\Omega_{\varepsilon}} p_{\varepsilon} \operatorname{div} \varphi_{\varepsilon} d x+\frac{\gamma}{\varepsilon^{4}} \int_{\Gamma_{\varepsilon}} u_{\varepsilon} \varphi_{\varepsilon} d \sigma\right) \\
& =\mu \int_{\Omega} \partial_{y_{3}} \tilde{u}^{\prime} \partial_{y_{3}} \tilde{\varphi}^{\prime} d y-\int_{\Omega} \tilde{p} \operatorname{div}_{y^{\prime}} \tilde{\varphi}^{\prime} d y+\gamma \int_{\Gamma} \tilde{u}^{\prime} \tilde{\varphi}^{\prime} d \sigma .
\end{aligned}
$$

(ii) If $\lambda \in(0,+\infty)$,

$$
\begin{aligned}
& \lim _{\varepsilon \rightarrow 0}\left(\frac{\mu}{\varepsilon^{3}} \int_{\Omega_{\varepsilon}} D u_{\varepsilon}: D \varphi_{\varepsilon} d x-\frac{1}{\varepsilon^{3}} \int_{\Omega_{\varepsilon}} p_{\varepsilon} \operatorname{div} \varphi_{\varepsilon} d x+\frac{\gamma}{\varepsilon^{4}} \int_{\Gamma_{\varepsilon}} u_{\varepsilon} \varphi_{\varepsilon} d \sigma\right) \\
& \quad=\mu \int_{\Omega} \partial_{y_{3}} \tilde{u}^{\prime} \partial_{y_{3}} \tilde{\varphi}^{\prime} d y-\int_{\Omega} \tilde{p} \operatorname{div}_{y^{\prime}} \tilde{\varphi}^{\prime} d y+\lambda^{2} \int_{\Gamma} R \tilde{u}^{\prime} \tilde{\varphi}^{\prime} d \sigma+\gamma \int_{\Gamma} \tilde{u}^{\prime} \tilde{\varphi}^{\prime} d \sigma .
\end{aligned}
$$

Proof. Taking $\varphi_{\varepsilon} / \varepsilon^{3}$ as a test function in (3.1), we have

$$
\begin{aligned}
& \frac{\mu}{\varepsilon^{3}} \int_{\Omega_{\varepsilon}} D u_{\varepsilon}: D \varphi_{\varepsilon} d x-\frac{1}{\varepsilon^{3}} \int_{\Omega_{\varepsilon}} p_{\varepsilon} \operatorname{div} \varphi_{\varepsilon} d x \\
& \quad+\frac{1}{\varepsilon^{3}} \int_{\Omega_{\varepsilon}}\left(u_{\varepsilon} \cdot \nabla\right) u_{\varepsilon} \varphi_{\varepsilon} d x+\frac{\gamma}{\varepsilon^{4}} \int_{\Gamma_{\varepsilon}} u_{\varepsilon} \varphi_{\varepsilon} d \sigma=\frac{1}{\varepsilon^{3}} \int_{\Omega_{\varepsilon}} f_{\varepsilon} \varphi_{\varepsilon} d x .
\end{aligned}
$$

Copyright (C) by SIAM. Unauthorized reproduction of this article is prohibited. 
The third term of this equality can be estimated by (3.3), (6.29), and (4.20) applied to $u_{\varepsilon}$ and $\varphi_{\varepsilon}$, which proves

$$
\frac{1}{\varepsilon^{3}} \int_{\Omega_{\varepsilon}}\left(u_{\varepsilon} \cdot \nabla\right) u_{\varepsilon} \varphi_{\varepsilon} d x=O_{\varepsilon}
$$

while the last term can be estimated by using the change of variables (3.5), (6.29), and (6.31), which easily gives

$$
\frac{1}{\varepsilon^{3}} \int_{\Omega_{\varepsilon}} f_{\varepsilon} \varphi_{\varepsilon} d x=\int_{\tilde{\Omega}_{\varepsilon}} \tilde{f} \frac{\tilde{\varphi} \varepsilon}{\varepsilon^{2}} d y=\int_{\Omega} \tilde{f}^{\prime} \tilde{\varphi}^{\prime} d y+O_{\varepsilon}
$$

So, (6.34) can be written as

$$
\frac{\mu}{\varepsilon^{3}} \int_{\Omega_{\varepsilon}} D u_{\varepsilon}: D \varphi_{\varepsilon} d x-\frac{1}{\varepsilon^{3}} \int_{\Omega_{\varepsilon}} p_{\varepsilon} \operatorname{div} \varphi_{\varepsilon} d x+\frac{\gamma}{\varepsilon^{4}} \int_{\Gamma_{\varepsilon}} u_{\varepsilon} \varphi_{\varepsilon} d \sigma=\int_{\Omega} \tilde{f}^{\prime} \tilde{\varphi}^{\prime} d y+O_{\varepsilon}
$$

Using now $\tilde{\varphi}$ as a test function in (3.10) and (3.12), (3.15), (3.16) depending on the value of $\lambda$ (for $\lambda=+\infty$, note that (6.29) and Lemma 5.4 imply $\tilde{\varphi} \in W^{\perp}$ on $\Gamma$ ), we then deduce (6.32) and (6.33) from (6.35).

Proof of Theorem 3.8. We divide the proof into four steps.

Step 1. We assume $\lambda=0,+\infty$; let us prove (3.29).

Thanks to Theorem 3.3 we can take $\varphi_{\varepsilon}=u_{\varepsilon}$ in Lemma 6.1. Using that $\operatorname{div} u_{\varepsilon}=0$ in $\Omega_{\varepsilon}$ and problem (3.10) plus (3.12) or (3.16) depending on the value of $\lambda$, we deduce

$$
\lim _{\varepsilon \rightarrow 0}\left(\frac{\mu}{\varepsilon^{3}} \int_{\Omega_{\varepsilon}}\left|D u_{\varepsilon}\right|^{2} d x+\frac{\gamma}{\varepsilon^{4}} \int_{\Gamma_{\varepsilon}}\left|u_{\varepsilon}\right|^{2} d \sigma\right)=\mu \int_{\Omega}\left|\partial_{y_{3}} \tilde{u}^{\prime}\right|^{2} d y+\gamma \int_{\Gamma}\left|\tilde{u}^{\prime}\right|^{2} d \sigma
$$

which, using on the left-hand side the change of variables (3.5), can be written as

$$
\begin{aligned}
& \lim _{\varepsilon \rightarrow 0}\left(\mu \int_{\Omega_{\varepsilon}}\left(\left|D_{y^{\prime}}\left(\frac{\tilde{u}_{\varepsilon}}{\varepsilon}\right)\right|^{2}+\left|\partial_{y_{3}}\left(\frac{\tilde{u}_{\varepsilon}}{\varepsilon^{2}}\right)\right|^{2}\right) d y+\gamma \int_{\Gamma}\left|\frac{\tilde{u}_{\varepsilon}}{\varepsilon^{2}}\right|^{2} d \sigma\right) \\
& \quad=\mu \int_{\Omega}\left|\partial_{y_{3}} \tilde{u}^{\prime}\right|^{2} d y+\gamma \int_{\Gamma}\left|\tilde{u}^{\prime}\right|^{2} d \sigma .
\end{aligned}
$$

Thanks to (3.8), this proves (3.29).

Step 2. We assume $\lambda \in(0,+\infty)$; let us prove that

$$
\begin{aligned}
E_{\varepsilon}:= & \frac{\mu}{\varepsilon^{3}} \int_{\Omega_{\varepsilon}^{-}}\left|D u_{\varepsilon}\right|^{2} d x \\
& +\frac{\mu}{\varepsilon^{3}} \int_{\Omega_{\varepsilon}^{+}}\left|D u_{\varepsilon}-\varepsilon \sum_{i=1}^{2} \partial_{y_{3}} \tilde{u}_{i}\left(x^{\prime}, \frac{x_{3}}{\varepsilon}\right) e_{i} \otimes e_{3}-\frac{\varepsilon^{\frac{3}{2}}}{r_{\varepsilon}^{\frac{1}{2}}} f_{C_{r_{\varepsilon}\left(x^{\prime}\right)}} D_{z} \widehat{u}\left(s^{\prime}, \frac{x}{r_{\varepsilon}}\right) d s^{\prime}\right|^{2} d x \\
& +\frac{\gamma}{\varepsilon^{4}} \int_{\Gamma_{\varepsilon}}\left|u_{\varepsilon}-\varepsilon^{2}\left(\tilde{u}^{\prime}\left(x^{\prime}, 0\right), 0\right)\right|^{2} d \sigma
\end{aligned}
$$

tends to zero, which in particular will imply (3.31). Developing the expression of $E_{\varepsilon}$, we have 


$$
\begin{aligned}
E_{\varepsilon}= & \frac{\mu}{\varepsilon^{3}} \int_{\Omega_{\varepsilon}}\left|D u_{\varepsilon}\right|^{2} d x+\frac{\gamma}{\varepsilon^{4}} \int_{\Gamma_{\varepsilon}}\left|u_{\varepsilon}\right|^{2} d \sigma+\frac{\mu}{\varepsilon} \int_{\Omega_{\varepsilon}^{+}}\left|\partial_{y_{3}} \tilde{u}^{\prime}\left(x^{\prime}, \frac{x_{3}}{\varepsilon}\right)\right|^{2} d x \\
& +\frac{\mu}{r_{\varepsilon}} \int_{\Omega_{\varepsilon}^{+}}\left|f_{C_{r_{\varepsilon}\left(x^{\prime}\right)}} D_{z} \widehat{u}\left(s^{\prime}, \frac{x}{r_{\varepsilon}}\right) d s^{\prime}\right|^{2} d x-\frac{2 \mu}{\varepsilon^{2}} \int_{\Omega_{\varepsilon}^{+}} \partial_{x_{3}} u_{\varepsilon}^{\prime} \partial_{y_{3}} \tilde{u}^{\prime}\left(x^{\prime}, \frac{x_{3}}{\varepsilon}\right) d x \\
& -\frac{2 \mu}{\varepsilon^{\frac{3}{2}} r_{\varepsilon}^{\frac{1}{2}}} \int_{\Omega_{\varepsilon}^{+}} f_{C_{r_{\varepsilon}\left(x^{\prime}\right)} D u_{\varepsilon}: D_{z} \widehat{u}\left(s^{\prime}, \frac{x}{r_{\varepsilon}}\right) d s^{\prime} d x} \\
& +\frac{2 \mu}{\varepsilon^{\frac{1}{2}} r_{\varepsilon}^{\frac{1}{2}}} \int_{\Omega_{\varepsilon}} \partial_{y_{3}} \tilde{u}^{\prime}\left(x^{\prime}, \frac{x_{3}}{\varepsilon}\right) f_{C_{r_{\varepsilon}\left(x^{\prime}\right)}} \partial_{z_{3}} \widehat{u}^{\prime}\left(s^{\prime}, \frac{x}{r_{\varepsilon}}\right) d s^{\prime} d x \\
& -\frac{2 \gamma}{\varepsilon^{2}} \int_{\Gamma_{\varepsilon}} u_{\varepsilon}^{\prime} \tilde{u}^{\prime}\left(x^{\prime}, 0\right) d \sigma+\gamma \int_{\Gamma_{\varepsilon}}\left|\tilde{u}^{\prime}\left(x^{\prime}, 0\right)\right|^{2} d \sigma .
\end{aligned}
$$

Let us pass to the limit in the different terms of this expression.

- First and second terms. They can be estimated taking $\varphi_{\varepsilon}=u_{\varepsilon}$ in (6.33), which gives

$$
\begin{aligned}
& \frac{\mu}{\varepsilon^{3}} \int_{\Omega_{\varepsilon}}\left|D u_{\varepsilon}\right|^{2} d x+\frac{\gamma}{\varepsilon^{4}} \int_{\Gamma_{\varepsilon}}\left|u_{\varepsilon}\right|^{2} d \sigma \\
& \quad=\mu \int_{\Omega}\left|\partial_{y_{3}} \tilde{u}^{\prime}\right|^{2} d y+\int_{\Gamma}\left(\gamma\left|\tilde{u}^{\prime}\right|^{2}+\lambda^{2} R \tilde{u}^{\prime} \tilde{u}^{\prime}\right) d \sigma+O_{\varepsilon} .
\end{aligned}
$$

- Third term. We use the change of variables (3.5), which gives

$$
\frac{\mu}{\varepsilon} \int_{\Omega_{\varepsilon}^{+}}\left|\partial_{y_{3}} \tilde{u}^{\prime}\left(x^{\prime}, \frac{x_{3}}{\varepsilon}\right)\right|^{2} d x=\mu \int_{\Omega}\left|\partial_{y_{3}} \tilde{u}^{\prime}\right|^{2} d y .
$$

- Fourth term. Using the change of variables (5.17), (6.22) and definition (3.14) of $R$, we get

$$
\begin{aligned}
& \frac{\mu}{r_{\varepsilon}} \int_{\Omega_{\varepsilon}^{+}}\left|f_{C_{r_{\varepsilon}\left(x^{\prime}\right)}} D_{z} \widehat{u}\left(s^{\prime}, \frac{x}{r_{\varepsilon}}\right) d s^{\prime}\right|^{2} d x=\mu \int_{\omega} \int_{\widehat{Q}_{\frac{\varepsilon}{r_{\varepsilon}}}}\left|f_{C_{\varepsilon}\left(x^{\prime}\right)} D_{z} \widehat{u}\left(s^{\prime}, z\right) d s^{\prime}\right|^{2} d z d x^{\prime} \\
& \quad=\mu \int_{\omega} \int_{\widehat{Q}}\left|D_{z} \widehat{u}\right|^{2} d z d x^{\prime}+O_{\varepsilon}=\lambda^{2} \int_{\Gamma} R \tilde{u}^{\prime} \tilde{u}^{\prime} d \sigma+O_{\varepsilon} .
\end{aligned}
$$

- Fifth term. The change of variables (3.5) together with (3.8) proves

$-\frac{2 \mu}{\varepsilon^{2}} \int_{\Omega_{\varepsilon}^{+}} \partial_{x_{3}} u_{\varepsilon}^{\prime} \partial_{y_{3}} \tilde{u}^{\prime}\left(x^{\prime}, \frac{x_{3}}{\varepsilon}\right) d x=-2 \mu \int_{\Omega} \partial_{y_{3}}\left(\frac{\tilde{u}_{\varepsilon}^{\prime}}{\varepsilon^{2}}\right) \partial_{y_{3}} \tilde{u}^{\prime} d y=-2 \mu \int_{\Omega}\left|\partial_{y_{3}} \tilde{u}^{\prime}\right|^{2} d y+O_{\varepsilon}$.

- Sixth term. We use the change of variables (5.17), statements (5.20), (6.22), and definition (3.14) of $R$, which gives

$$
\begin{aligned}
& -\frac{2 \mu}{\varepsilon^{\frac{3}{2}} r_{\varepsilon}^{\frac{1}{2}}} \int_{\Omega_{\varepsilon}^{+}} \int_{C_{r_{\varepsilon}\left(x^{\prime}\right)}} D u_{\varepsilon}: D_{z} \widehat{u}\left(s^{\prime}, \frac{x}{r_{\varepsilon}}\right) d s^{\prime} d x=-2 \mu \int_{\omega} \int_{\widehat{Q}_{\frac{\varepsilon}{r_{\varepsilon}}}} D_{z} \widehat{u}_{\varepsilon}: D_{z} \widehat{u} d z d s^{\prime} \\
& =-2 \mu \int_{\omega} \int_{\widehat{Q}}\left|D_{z} \widehat{u}\right|^{2} d z d s^{\prime}+O_{\varepsilon}=-2 \lambda^{2} \int_{\Gamma} R \tilde{u}^{\prime} \tilde{u}^{\prime} d \sigma+O_{\varepsilon} .
\end{aligned}
$$

Copyright $@$ by SIAM. Unauthorized reproduction of this article is prohibited. 
- Seventh term. We consider $s_{\varepsilon}>0$ such that

$$
\lim _{\varepsilon \rightarrow 0} \frac{s_{\varepsilon}}{\varepsilon}=0, \quad \lim _{\varepsilon \rightarrow 0} \frac{s_{\varepsilon}}{r_{\varepsilon}}=+\infty .
$$

Then we remark that the change of variables (3.5) gives

$$
\frac{1}{\varepsilon} \int_{\left\{0<x_{3}<s_{\varepsilon}\right\}}\left|\partial_{y_{3}} \tilde{u}^{\prime}\left(x^{\prime}, \frac{x_{3}}{\varepsilon}\right)\right|^{2} d x=\int_{\left\{0<y_{3}<\frac{s_{\varepsilon}}{\varepsilon}\right\}}\left|\partial_{y_{3}} \tilde{u}^{\prime}\right|^{2} d y=O_{\varepsilon},
$$

while the change of variables (5.17) and the Cauchy-Schwarz inequality prove

$$
\begin{aligned}
& \frac{1}{r_{\varepsilon}} \int_{\left\{s_{\varepsilon}<x_{3}\right\}}\left|f_{C_{r_{\varepsilon}\left(x^{\prime}\right)}} \partial_{z_{3}} \widehat{u}^{\prime}\left(s^{\prime}, \frac{x}{r_{\varepsilon}}\right) d s^{\prime}\right|^{2} d x \\
& \quad=r_{\varepsilon}^{2} \sum_{k^{\prime} \in \mathbb{Z}^{2}} \int_{Z^{\prime}} \int_{\frac{s_{\varepsilon}}{r_{\varepsilon}}}^{\frac{\varepsilon}{r_{\varepsilon}}}\left|f_{C_{r_{\varepsilon}}^{k^{\prime}}} \partial_{z_{3}} \widehat{u}^{\prime}\left(s^{\prime}, z\right) d s^{\prime}\right|^{2} d z_{3} d z^{\prime} \\
& \quad \leq \int_{\omega} \int_{Z^{\prime}} \int_{\frac{s_{\varepsilon}}{r_{\varepsilon}}}^{\infty}\left|\partial_{z_{3}} \widehat{u}^{\prime}\left(s^{\prime}, z\right)\right|^{2} d z_{3} d z^{\prime} d s^{\prime}=O_{\varepsilon} .
\end{aligned}
$$

From (6.38) and (6.39), we get

$$
\frac{2 \mu}{\varepsilon^{\frac{1}{2}} r_{\varepsilon}^{\frac{1}{2}}} \int_{\Omega_{\varepsilon}} \partial_{y_{3}} \tilde{u}^{\prime}\left(x^{\prime}, \frac{x_{3}}{\varepsilon}\right) f_{C_{r_{\varepsilon}\left(x^{\prime}\right)}} \partial_{z_{3}} \widehat{u}^{\prime}\left(s^{\prime}, \frac{x}{r_{\varepsilon}}\right) d s^{\prime} d x=O_{\varepsilon} .
$$

- Eighth term. Using

$$
\int_{\Gamma_{\varepsilon}}\left|u_{\varepsilon}-u_{\varepsilon}\left(x^{\prime}, 0\right)\right|^{2} d x=O_{\varepsilon}
$$

and (3.8), we have

$$
-\frac{2 \gamma}{\varepsilon^{2}} \int_{\Gamma_{\varepsilon}} u_{\varepsilon}^{\prime} \tilde{u}^{\prime}\left(x^{\prime}, 0\right) d \sigma=-2 \gamma \int_{\Gamma} \frac{\tilde{u}_{\varepsilon}^{\prime}}{\varepsilon^{2}} \tilde{u}^{\prime} d \sigma+O_{\varepsilon}=-2 \gamma \int_{\Gamma}\left|\tilde{u}^{\prime}\right|^{2} d \sigma+O_{\varepsilon} .
$$

- Ninth term. We have

$$
\gamma \int_{\Gamma_{\varepsilon}}\left|\tilde{u}^{\prime}\left(x^{\prime}, 0\right)\right|^{2} d \sigma=\gamma \int_{\Gamma}\left|\tilde{u}^{\prime}\right|^{2} d \sigma+O_{\varepsilon}
$$

The estimates obtained for the different terms on the right-hand side of (6.36) prove that $E_{\varepsilon}$ tends to zero and then (3.31).

Step 3. Let us now prove that (3.28) holds.

By Corollary 4.3 and (3.3), for every $\varepsilon>0$ there exists $\phi_{\varepsilon} \in H_{0}^{1}\left(\Omega_{\varepsilon}\right)^{3}$ satisfying

$$
\operatorname{div} \phi_{\varepsilon}=p_{\varepsilon} \text { in } \Omega_{\varepsilon}, \quad\left\|\phi_{\varepsilon}\right\|_{H_{0}^{1}\left(\Omega_{\varepsilon}\right)^{3}} \leq \frac{C}{\sqrt{\varepsilon}} \quad \forall \varepsilon>0 .
$$

Applying Lemma 5.1 to the sequence $\varphi_{\varepsilon}=\varepsilon^{2} \phi_{\varepsilon}$ and taking into account (3.9) and that $\phi_{\varepsilon}=0$ on $\Gamma_{\varepsilon}$, we deduce that, up to a subsequence, there exist $\tilde{\phi}^{\prime} \in H^{1}\left(0,1 ; L^{2}(\omega)\right)^{2}$ and $\tilde{\psi} \in H^{1}\left(0,1 ; H^{-1}(\omega)\right)$ satisfying

$$
\begin{gathered}
\tilde{\phi}^{\prime}(0)=\tilde{\phi}^{\prime}(1)=0 \text { in } L^{2}(\omega), \quad \tilde{\psi}(0)=\tilde{\psi}(1)=0 \text { in } H^{-1}(\omega), \\
\operatorname{div}_{y^{\prime}} \tilde{\phi}^{\prime}+\partial_{y_{3}} \tilde{\psi}=\tilde{p} \text { in } H^{1}\left(0,1 ; H^{-1}(\omega)\right),
\end{gathered}
$$

Copyright (c) by SIAM. Unauthorized reproduction of this article is prohibited. 
such that defining $\tilde{\phi}_{\varepsilon} \in H_{0}^{1}\left(\tilde{\Omega}_{\varepsilon}\right)^{3}$ by

$$
\tilde{\phi}_{\varepsilon}(y)=\phi_{\varepsilon}\left(y^{\prime}, \varepsilon y_{3}\right), \text { a.e. } y \in \tilde{\Omega}_{\varepsilon},
$$

we have

$$
\begin{gathered}
\varepsilon \tilde{\phi}_{\varepsilon} \rightarrow 0 \text { in } H^{1}(\Omega)^{3}, \quad \tilde{\phi}_{\varepsilon} \rightarrow\left(\tilde{\phi}^{\prime}, 0\right) \text { in } H^{1}\left(0,1 ; L^{2}(\omega)\right)^{3}, \\
\frac{\tilde{\phi}_{\varepsilon, 3}}{\varepsilon} \rightarrow \tilde{\psi} \text { in } H^{1}\left(0,1 ; H^{-1}(\omega)\right), \quad \operatorname{div}_{y^{\prime}} \tilde{\phi}_{\varepsilon}^{\prime}+\frac{1}{\varepsilon} \partial_{y_{3}} \tilde{\phi}_{\varepsilon, 3} \rightarrow \tilde{p} \text { in } L^{2}(\Omega) .
\end{gathered}
$$

Applying (6.32), (6.33) to the sequence $\varphi_{\varepsilon}=\varepsilon^{2} \phi_{\varepsilon}$ and using $\tilde{\phi}^{\prime}=0$ on $\Gamma$ and (6.40), we have

$$
\begin{aligned}
& \lim _{\varepsilon \rightarrow 0}\left(\frac{\mu}{\varepsilon} \int_{\Omega_{\varepsilon}} D u_{\varepsilon}: D \phi_{\varepsilon} d x-\frac{1}{\varepsilon} \int_{\Omega_{\varepsilon}}\left|p_{\varepsilon}\right|^{2} d x\right) \\
& \quad=\mu \int_{\Omega} \partial_{y_{3}} \tilde{u}^{\prime} \partial_{y_{3}} \tilde{\phi}^{\prime} d y-\int_{\Omega} \tilde{p} \operatorname{div}_{y^{\prime}} \tilde{\phi}^{\prime} d y \quad \forall \lambda \in[0,+\infty] .
\end{aligned}
$$

Since $\tilde{p}$ does not depend on $y_{3}$, we deduce from (6.41) that

$$
\int_{\Omega} \tilde{p} \partial_{y_{3}} \tilde{\psi} d y=0
$$

and then by (6.41), the last term in (6.43) can be written as

$$
\int_{\Omega} \tilde{p} \operatorname{div}_{y^{\prime}} \tilde{\phi}^{\prime} d y=\int_{\omega}|\tilde{p}|^{2} d y^{\prime} .
$$

Now, we reason depending on the value of $\lambda$.

If $\lambda=0$ or $+\infty$, we use that (3.29), (6.40), and (6.42) imply

$$
\lim _{\varepsilon \rightarrow 0} \frac{1}{\varepsilon} \int_{\Omega_{\varepsilon}} D u_{\varepsilon}: D \phi_{\varepsilon} d x=\int_{\Omega} \partial_{y_{3}} \tilde{u}^{\prime} \partial_{y_{3}} \tilde{\phi}^{\prime} d y,
$$

and thus, by (6.43), (6.44), we have

$$
\lim _{\varepsilon \rightarrow 0} \frac{1}{\varepsilon} \int_{\Omega_{\varepsilon}}\left|p_{\varepsilon}\right|^{2} d y=\int_{\omega}|\tilde{p}|^{2} d y^{\prime} .
$$

Using the change of variables (3.5) and that $\tilde{p}_{\varepsilon}$ converges weakly to $\tilde{p}$ in $L^{2}(\Omega)$, this easily proves (3.28).

If $\lambda \in(0,+\infty)$, we apply Lemma 5.4 to $\varepsilon^{2} \phi_{\varepsilon}$ at the place of $u_{\varepsilon}$, which combined with $\tilde{\phi}^{\prime}=0$ on $\Gamma$ proves, up to a subsequence, the existence of $\widehat{\phi} \in L^{2}\left(\Omega ; \mathcal{V}^{3}\right)$, with

$$
\widehat{\phi}_{3}\left(x^{\prime}, z^{\prime}, 0\right)=-\lambda \nabla \Psi\left(z^{\prime}\right) \tilde{\phi}^{\prime}\left(x^{\prime}, 0\right)=0, \quad \text { a.e. }\left(x^{\prime}, z^{\prime}\right) \in \omega \times Z^{\prime},
$$

such that the sequence $\widehat{\phi}_{\varepsilon} \in L^{2}\left(\omega ; H^{1}\left(\omega \times \widehat{Z}_{\varepsilon}\right)\right)^{3}$ defined by

$$
\widehat{\phi}_{\varepsilon}\left(x^{\prime}, z\right)=\phi_{\varepsilon}\left(r_{\varepsilon} \kappa\left(\frac{x^{\prime}}{r_{\varepsilon}}\right)+r_{\varepsilon} z^{\prime}, r_{\varepsilon} z_{3}\right), \quad \text { a.e. }\left(x^{\prime}, z\right) \in \omega \times \widehat{Z}_{\varepsilon}
$$

satisfies 


$$
\frac{\varepsilon^{\frac{1}{2}}}{r_{\varepsilon}^{\frac{1}{2}}} D_{z} \widehat{\phi}_{\varepsilon} \rightarrow D_{z} \widehat{\phi} \text { in } L^{2}\left(\omega \times \widehat{Q}_{M}\right)^{3} \quad \forall M>0 .
$$

Using (3.31), (6.40), the changes of variables (3.5), (5.17), and the convergences (6.42), (6.47), we deduce

$$
\begin{aligned}
& \frac{1}{\varepsilon} \int_{\Omega_{\varepsilon}} D u_{\varepsilon}: D \phi_{\varepsilon} d x=\int_{\Omega} \partial_{y_{3}} \tilde{u}^{\prime} \partial_{y_{3}} \tilde{\phi}_{\varepsilon}^{\prime} d y+\frac{\varepsilon^{\frac{1}{2}}}{r_{\varepsilon}^{\frac{1}{2}}} \int_{\omega \times \widehat{Q}_{\frac{\varepsilon}{r_{\varepsilon}}}} D_{z} \widehat{u}: D_{z} \widehat{\phi}_{\varepsilon} d x^{\prime} d z+O_{\varepsilon} \\
& \quad=\int_{\Omega} \partial_{y_{3}} \tilde{u}^{\prime} \partial_{y_{3}} \tilde{\phi}^{\prime} d y+\int_{\omega \times \widehat{Q}} D_{z} \widehat{u}: D_{z} \widehat{\phi} d x^{\prime} d z+O_{\varepsilon}
\end{aligned}
$$

Therefore, (6.43), (6.44) give

$$
\lim _{\varepsilon \rightarrow 0} \frac{1}{\varepsilon} \int_{\Omega_{\varepsilon}}\left|p_{\varepsilon}\right|^{2} d x=\int_{\omega}|\tilde{p}|^{2} d x^{\prime}+\mu \int_{\omega \times \widehat{Q}} D_{z} \widehat{u}: D_{z} \widehat{\phi} d x^{\prime} d z
$$

On the other hand, using the change of variables (5.17), and taking into account (6.40), (3.3), we have

$$
\int_{\omega} \int_{\widehat{Z}_{\varepsilon}}\left|\frac{\varepsilon^{\frac{1}{2}}}{r_{\varepsilon}^{\frac{1}{2}}} \operatorname{div}_{z} \widehat{\phi}_{\varepsilon}\right|^{2} d z d x^{\prime}=\int_{\Omega_{\varepsilon}}\left|\operatorname{div} \phi_{\varepsilon}\right|^{2} d x \leq C \varepsilon^{2}
$$

and thus by $(6.47)$ we deduce

$$
\operatorname{div}_{z} \widehat{\phi}=0 \quad \text { in } \omega \times \widehat{Q}
$$

By (6.46) and (6.49), we can take $\widehat{\phi}$ as a test function in (6.21) to obtain

$$
\int_{\omega \times \widehat{Q}} D_{z} \widehat{u}: D_{z} \widehat{\phi} d x^{\prime} d z=0
$$

Therefore (6.48) proves that (6.45) also holds in the case $\lambda \in(0,+\infty)$, which allows us to conclude (3.28) as above.

Step 4. In order to finish the proof of Theorem 3.8, it remains to show (3.27).

We consider a sequence $s_{\varepsilon}>0$ satisfying (6.37). Using that $u_{\varepsilon}$ and $\tilde{u}^{\prime}$ vanish on $\omega \times\{\varepsilon\}$ and $\omega \times\{1\}$, respectively, and taking into account (3.3), (3.29), (3.31) and, in the case $\lambda \in(0,+\infty),(6.39)$, we easily get

Copyright (c) by SIAM. Unauthorized reproduction of this article is prohibited. 


$$
\begin{aligned}
& \frac{1}{\varepsilon^{5}} \int_{\Omega_{\varepsilon}^{-}}\left|u_{\varepsilon}\right|^{2} d x+\frac{1}{\varepsilon^{5}} \int_{\Omega_{\varepsilon}^{+}}\left(\left|u_{\varepsilon, 3}\right|^{2}+\left|u_{\varepsilon}^{\prime}-\varepsilon^{2} \tilde{u}^{\prime}\left(x^{\prime}, \frac{x_{3}}{\varepsilon}\right)\right|^{2}\right) d x \\
& \leq \frac{2}{\varepsilon^{5}} \int_{\Omega_{\varepsilon} \cap\left\{x_{3}<s_{\varepsilon}\right\}}\left|u_{\varepsilon}\right|^{2} d x+\frac{2}{\varepsilon} \int_{\Omega \cap\left\{x_{3}<s_{\varepsilon}\right\}}\left|\tilde{u}^{\prime}\left(x^{\prime}, \frac{x_{3}}{\varepsilon}\right)\right|^{2} d x \\
&+\frac{1}{\varepsilon^{5}} \int_{\left\{x_{3}>s_{\varepsilon}\right\}}\left(\left|u_{\varepsilon, 3}\right|^{2}+\left|u_{\varepsilon}^{\prime}-\varepsilon^{2} \tilde{u}^{\prime}\left(x^{\prime}, \frac{x_{3}}{\varepsilon}\right)\right|^{2}\right) d x \\
& \leq \frac{2}{\varepsilon^{5}} \int_{\omega} \int_{-\delta_{\varepsilon} \psi\left(\frac{x^{\prime}}{\varepsilon}\right)}^{s_{\varepsilon}}\left|\int_{x_{3}}^{\varepsilon} \partial_{x_{3}} u_{\varepsilon}\left(x^{\prime}, t\right) d t\right|^{2} d x_{3} d x^{\prime} \\
&+\frac{2}{\varepsilon^{3}} \int_{\omega} \int_{0}^{s_{\varepsilon}}\left|\int_{x_{3}}^{\varepsilon} \partial_{y_{3}} \tilde{u}^{\prime}\left(x^{\prime}, \frac{t}{\varepsilon}\right) d t\right|^{2} d x_{3} d x^{\prime} \\
&+\frac{1}{\varepsilon^{5}} \int_{\omega} \int_{s_{\varepsilon}}^{\varepsilon}\left(\left|\int_{x_{3}}^{\varepsilon} \partial_{x_{3}} u_{\varepsilon, 3}\left(x^{\prime}, t\right) d t\right|^{2}\right. \\
&\left.+\left|\int_{x_{3}}^{\varepsilon}\left(\partial_{x_{3}} u_{\varepsilon}^{\prime}\left(x^{\prime}, t\right)-\varepsilon \partial_{y_{3}} \tilde{u}^{\prime}\left(x^{\prime}, \frac{t}{\varepsilon}\right)\right) d t\right|^{2}\right) d x_{3} d x^{\prime} \\
& \leq \frac{2\left(s_{\varepsilon}+C \delta_{\varepsilon}\right)}{\varepsilon^{4}} \int_{\Omega_{\varepsilon}}\left|\partial_{x_{3}} u_{\varepsilon}\right|^{2} d x+\frac{2 s_{\varepsilon}}{\varepsilon} \int_{\Omega}\left|\partial_{y_{3}} \tilde{u}^{\prime}\right|^{2} d y \\
&+ \frac{1}{\varepsilon^{3}} \int_{\left\{x_{3}>s_{\varepsilon}\right\}}\left(\left|\partial_{x_{3}} u_{\varepsilon, 3}\right|^{2}+\left|\partial_{x_{3}} u_{\varepsilon}^{\prime}-\varepsilon \partial_{y_{3}} \tilde{u}^{\prime}\left(x^{\prime}, \frac{x_{3}}{\varepsilon}\right)\right|^{2}\right) d x=O_{\varepsilon} .
\end{aligned}
$$

This proves (3.27).

Acknowledgment. The authors thank Didier Bresch for interesting discussions and many suggestions concerning this paper.

\section{REFERENCES}

[1] G. Allaire, Homogenization and two-scale convergence, SIAM J. Math. Anal., 23 (1992), pp. $1482-1518$.

[2] Y. Amirat, D. Bresch, J. Lemoine, and J. Simon, Effect of rugosity on a flow governed by stationary Navier-Stokes equations, Quart. Appl. Math., 59 (2001), pp. 769-785.

[3] Y. Amirat, B. Climent, E. Fernández-CARA, and J. Simon, The Stokes equations with Fourier boundary conditions on a wall with asperities, Math. Models Methods Appl. Sci., 24 (2001), pp. 255-276.

[4] T. Arbogast, J. Douglas, JR., And U. Hornung, Derivation of the double porosity model of single phase flow via homogenization theory, SIAM J. Math. Anal., 21 (1990), pp. 823-836.

[5] G. Bayada And M. Chambat, Homogenization of the Stokes system in a thin film flow with rapidly varying thickness, RAIRO Model. Math. Anal. Numer., 23 (1989), pp. 205-234.

[6] G. Bayada And M. Chambat, New models in the theory of the hydrodynamic lubrication of rough surfaces, Trans. Amer. Math. Soc. J. Trib., 110 (1988), pp. 402-407.

[7] N. Benhaboucha, M. Chambat, And I. Ciuperca, Asymptotic behaviour of pressure and stresses in a thin film flow with a rough boundary, Quart. Appl. Math., 63 (2005), pp. 369-400.

[8] D. Bresch, C. Choquet, L. Chupin, T. Colin, and M. Gisclon, Roughness-induced effect at main order on the Reynolds approximation, Multiscale Model. Simul., 8 (2010), pp. 997-1017.

[9] D. Bucur, E. Feireisl, And S. NeČAsová, Boundary behavior of viscous fluids: Influence of wall roughness and friction-driven boundary conditions, Arch. Rational Mech. Anal., 197 (2010), pp. 117-138.

[10] D. Bucur, E. Feireisl, S. NeČasová, And J. Wolf, On the asymptotic limit of the Navier-Stokes system on domains with rough boundaries, J. Differential Equations, 244 (2008), pp. 2890-2908.

Copyright $@$ ㅇ by SIAM. Unauthorized reproduction of this article is prohibited. 
[11] J. Casado-Díaz, Two-scale convergence for nonlinear Dirichlet problems in perforated domains, Proc. Roy. Soc. Edinburgh Sect. A, 130 (2000), pp. 249-276.

[12] J. Casado-Díaz, E. Fernández-Cara, And J. Simon, Why viscous fluids adhere to rugose walls: A mathematical explanation, J. Differential Equations, 189 (2003), pp. 526-537.

[13] J. Casado-Díaz, M. Luna-Laynez, and J. D. Martín-Gómez, An adaptation of the multiscale methods for the analysis of very thin reticulated structures, C. R. Acad. Sci. Paris Sér. I, 332 (2001), pp. 223-228.

[14] J. Casado-Díaz, M. Luna-Laynez, and F. J. SuÁRez-Grau, Asymptotic behavior of a viscous fluid with slip boundary conditions on a slightly rough wall, Math. Models Methods Appl. Sci., 20 (2010), pp. 121-156.

[15] J. Casado-Díaz, M. Luna-Laynez, and F. J. SuÁrez-Grau, A viscous fluid in a thin domain satisfying the slip condition on a slightly rough boundary, C. R. Acad. Sci. Paris Ser. I, 348 (2010), pp. 967-971.

[16] J. Casado-Díaz, M. Luna-Laynez, and F. J. Suárez-Grau, Estimates for the asymptotic expansion of a viscous fluid satisfying Navier's law on a rugous boundary, Math. Methods Appl. Sci., 34 (2011), pp. 1553-1561.

[17] J. Casado-Díaz, M. Luna-Laynez, and F. J. Suárez-Grau, The homogenization of elliptic partial differential systems on rugous domains with variable boundary conditions, Proc. Roy. Soc. Edinburgh, 143 (2013), pp. 303-335.

[18] D. Cioranescu, A. Damlamian, And G. Griso, Periodic unfolding and homogenization, C. R. Acad. Sci. Paris Sér. I, 335 (2002), pp. 99-104.

[19] W. JÄGER AND A. Mikelić, Couette flows over a rough boundary and drag reduction, Comm. Math. Phys., 232 (2003), pp. 429-455.

[20] M. Lenczner, Homogénéisation d'un circuit électrique, C. R. Acad. Sci. Paris Sér. II, 324 (1997), pp. 537-542.

[21] J.-L. Lions, Quelques méthodes de résolution des problèmes aux limites non linéaires, Dunod, Gauthier-Villars, Paris, 1969.

[22] G. NGuetseng, A general convergence result for a functional related to the theory of homogenization, SIAM J. Math. Anal., 20 (1989), pp. 608-623.

[23] L. Tartar, Topics in Nonlinear Analysis, Pub. Math. Orsay 78 13, Université de Paris-Sud, Département de Mathématique, Orsay, 1978.

[24] R. Temam, Navier-Stokes Equations and Nonlinear Functional Analysis, CBMS-NSF Regional Conf. Ser. in Appl. Math., 41, SIAM, Philadelphia, 1983.

Copyright (c) by SIAM. Unauthorized reproduction of this article is prohibited. 